\title{
Corner treatments for high-order local absorbing boundary conditions in high-frequency acoustic scattering*
}

\author{
A. Modave ${ }^{1}$, C. Geuzaine ${ }^{2}$, and X. Antoine ${ }^{3}$ \\ ${ }^{1}$ Laboratoire POEMS, CNRS, Inria, ENSTA Paris, Institut Polytechnique de Paris, 91120 Palaiseau, France, \\ axel.modave@ensta-paris.fr \\ ${ }^{2}$ Université de Liège, Institut Montefiore B28, 4000 Liège, Belgium, cgeuzaine@uliege.be \\ ${ }^{3}$ Institut Elie Cartan de Lorraine, Université de Lorraine, Inria Nancy-Grand Est EPI SPHINX, 54506 \\ Vandoeuvre-lès-Nancy Cedex, France, xavier.antoine@univ-lorraine.fr
}

\begin{abstract}
This paper deals with the design and validation of accurate local absorbing boundary conditions set on convex polygonal and polyhedral computational domains for the finite element solution of highfrequency acoustic scattering problems. While high-order absorbing boundary conditions (HABCs) are accurate for smooth fictitious boundaries, the precision of the solution drops in the presence of corners if no specific treatment is applied. We present and analyze two strategies to preserve the accuracy of Padé-type HABCs at corners: first by using compatibility relations (derived for right angle corners) and second by regularizing the boundary at the corner. Exhaustive numerical results for two- and three-dimensional problems are reported in the paper. They show that using the compatibility relations is optimal for domains with right angles. For the other cases, the error still remains acceptable, but depends on the choice of the corner treatment according to the angle.
\end{abstract}

\section{Introduction}

Large-scale high-frequency scattering problems need to be solved in many application areas, such as seismic and medical imaging, aeroacoustics, seismic risk assessment and electromagnetic compatibility. When specific frequencies of interest are identified, time-harmonic solvers are conveniently considered. Unfortunately, because of the highly oscillatory nature of the wave fields, these solvers lead to discretizations with a large number of unknowns and require the solution of large poorly-conditioned linear systems. Research on accurate and computationally efficient methods is very active: we can mention for instance recent works on high-frequency boundary element methods [7, 21, 22], high-order finite element methods [14, 16, 57, 68] and domain decomposition methods [9, 10, 34, 77].

Finite element methods are suited to realistic settings and complicated geometries, thanks to unstructured meshes and robust formulations. For solving scattering problems, they must be coupled with domain truncation techniques, which simulate the outward propagation of waves at the boundary of the computational domain. Thereby, couplings have been proposed with exact non-local boundary conditions [54], local absorbing boundary conditions (e.g. [5, 8, 53, 62, 70, 72, 73]) and perfectly marched layers (e.g. [17, 50, 67, 74]). In particular, high-order absorbing boundary conditions (HABCs) and perfectly matched layers (PMLs) provide high-fidelity solutions for limited computational costs, which makes them attractive techniques for large-scale simulations. Mostly because PMLs are easier to use, they have received much more attention than HABCs. Nevertheless, the parameter selection of PMLs remains tricky and critically depends on the discretization scheme $[17,48,66]$. By contrast, a priori analyses allow for a better control of the error, and make the parameter selection of HABCs easier $[15,44,47]$.

In the present paper, we are interested in applying Padé-type HABCs to computational domains with non-regular boundaries. Such HABCs have proved to be very efficient for planar boundaries [31] and

\footnotetext{
${ }^{*}$ Published in Journal of Computational Physics.
} 
regular curved boundaries $[4,8,53]$. However, difficulties arise when they are prescribed on non-regular boundaries with corners, such as the boundaries of polygonal and polyhedral domains. Specific corner treatments have been proposed for classical low-order absorbing boundary conditions [13, 51] and some HABCs [23, 43, 56, 71, 75] in settings with only right angles. These treatments rely on compatibility relations and involve, in some cases, auxiliary unknowns defined at the corners. Dealing with non-right angles and general-shaped non-regular boundaries is much more challenging, whatever boundary truncation technique is considered. Few corner treatments have been tested for specific boundary truncation techniques $[27,39,52]$ and, to the best of our knowledge, only in two dimensions with polygonal domains.

Here, we derive and analyze two approaches for applying Padé-type HABCs to Helmholtz problems with computational domains having right and non-right angles in two and three dimensions. These approaches are based on compatibility relations (derived for settings with right angles) and a regularization of the boundary. They can offer geometric flexibility when choosing the computational domain in application contexts, eventually leading to more appropriate shapes and smaller computational costs. Let us mention that the Padé-type HABCs are also used in acceleration techniques for boundary integral solvers $[6,21,26]$ and domain decomposition methods [18]. The analysis proposed here could also improve these acceleration techniques, which is currently investigated.

The Padé-type HABC for planar boundaries is derived in Section 2. Section 3 is dedicated to corner and edge treatments for settings with right angles. These treatments are based on compatibility relations, which are derived for two and three dimensions. The parameter selection is discussed and the effectiveness of the compatibility relations is asserted with finite element results. In section 4, we present two families of approximate approaches to deal with corners and edges with non-right angles. These approaches are evaluated and compared thanks to numerical results in two and three dimensions. It appears that the compatibility relations are optimal for settings with right angles. For angles lower than $2 \pi / 3$, using these relations still provides the best results, leading to acceptable levels of error. For more obtuse angles, it is then preferable to use regularization-based approaches which are more accurate. Finally, a conclusion and some perspectives are proposed in section 5 .

\section{Padé-type HABC for planar boundary}

In this section, we derive a family of high-order absorbing boundary conditions (HABCs) for planar boundaries. For the derivation, we consider the Helmholtz problem

$$
\Delta v+\kappa^{2} v=s, \quad \text { in } \mathbb{R}^{3},
$$

with the wavenumber $\kappa(x, y, z)$ and the source term $s(x, y, z)$. The unbounded domain $\mathbb{R}^{3}$ is decomposed into the interior region $\Omega=\left\{(x, y, z) \in \mathbb{R}^{3}: x<0\right\}$ and the exterior region $\Omega^{\text {ext }}=\left\{(x, y, z) \in \mathbb{R}^{3}: x>0\right\}$ separated by the plane interface $\Gamma$ with the equation $\{x=0\}$. The exterior medium is assumed to be homogeneous ( $\kappa$ is constant in $\Omega^{\text {ext }}$ ) and free of source (the support of $s$ is compact on $\Omega$ ). We seek to prescribe a boundary condition on $\Gamma$ to represent the outward propagation of waves leaving $\Omega$.

\subsection{Exact nonreflecting boundary condition}

The exact nonreflecting boundary condition is classically obtained by solving the exterior Helmholtz problem defined in $\Omega^{\text {ext }}$ for some Dirichlet data $\bar{v}(y, z)$ on $\Gamma$ [31]. Applying the multidimensional transverse Fourier transform $\mathcal{F}_{y z}$ in the $y$ - and $z$-directions to the Helmholtz equation yields

$$
\left(\partial_{x}^{2}+\lambda_{+} \lambda_{-}\right) \mathcal{F}_{y z}[v]\left(x, \xi_{y}, \xi_{z}\right)=0, \quad \text { for } x>0, \xi_{y} \in \mathbb{R}, \xi_{z} \in \mathbb{R}
$$

where $\xi_{y}$ and $\xi_{z}$ are the dual variables of $y$ and $z$ in the Fourier space, and the symbols $\lambda_{+}$and $\lambda_{-}$are defined as

$$
\lambda_{ \pm}\left(\xi_{y}, \xi_{z}\right) \equiv \pm \imath \sqrt{\kappa^{2}-\xi_{y}^{2}-\xi_{z}^{2}}
$$

The solution that contains only outgoing traveling modes and bounded evanescent modes reads

$$
\mathcal{F}_{y z}[v]\left(x, \xi_{y}, \xi_{z}\right)=\mathcal{F}_{y z}[\bar{v}]\left(\xi_{y}, \xi_{z}\right) e^{x \lambda_{+}\left(\xi_{y}, \xi_{z}\right)}
$$


Taking the derivative in $x$ of this solution leads to

$$
\partial_{x} \mathcal{F}_{y z}[v]\left(x, \xi_{y}, \xi_{z}\right)=\lambda_{+}\left(\xi_{y}, \xi_{z}\right) \mathcal{F}_{y z}[v]\left(x, \xi_{y}, \xi_{z}\right),
$$

and then, using the inverse Fourier transform $\mathcal{F}_{y z}^{-1}$,

$$
\partial_{x} v(x, y, z)=\mathcal{F}_{y z}^{-1}\left[\lambda_{+}\left(\xi_{y}, \xi_{z}\right) \mathcal{F}_{y z}[v]\left(x, \xi_{y}, \xi_{z}\right)\right] .
$$

Taking the restriction on $\Gamma$ gives the exact nonreflective boundary condition for the interior problem,

$$
\left.\partial_{x} v\right|_{\Gamma}=\left.\mathcal{B} v\right|_{\Gamma}, \quad \text { on } \Gamma
$$

where $\mathcal{B}$ is the pseudo-differential operator defined as

$$
\mathcal{B} \equiv \mathcal{F}_{y z}^{-1}\left[\lambda_{+}\left(\xi_{y}, \xi_{z}\right) \mathcal{F}_{y z}\right]=\imath \kappa \sqrt{1+\Delta_{\Gamma} / \kappa^{2}}
$$

with the Laplace-Beltrami operator $\Delta_{\Gamma}=\Delta-\partial_{x x}$. The operator $\mathcal{B}$ is the exact Dirichlet-to-Neumann $(\mathrm{DtN})$ operator for this problem. Unfortunately, because of the square root in the symbol of $\mathcal{B}$, the boundary condition is non-local. When using a finite element solver, it then leads to a strong coupling of the unknowns defined onto the fictitious boundary, and then to a dense block in the matrix of the linear system for the corresponding entries.

\subsection{Rational approximation of the square root $\sqrt{1+X}$}

Local boundary conditions can be derived by approximating the square root in the symbol of the DtN operator (1). In their seminal paper, Engquist and Majda [31] derived a family of local boundary conditions by using a Padé approximation of the square root. The $(2 N+1)^{\text {th }}$-order Padé approximation of the square root $f(X)=\sqrt{1+X}$ is classically written as the rational function $[12,38]$

$$
f_{2 N+1}^{\text {Padé }}(X)=1+\frac{2}{M} \sum_{i=1}^{N} \frac{a_{i} X}{1+b_{i} X},
$$

which we rewrite as

$$
f_{2 N+1}^{\text {Padé }}(X)=1+\frac{2}{M} \sum_{i=1}^{N} c_{i}\left(1-\frac{c_{i}+1}{c_{i}+1+X}\right)
$$

with $a_{i}=\sin ^{2}(i \pi / M), b_{i}=\cos ^{2}(i \pi / M), c_{i}=\tan ^{2}(i \pi / M)$ and $M=2 N+1$. Unfortunately, Padé approximations lead to boundary conditions that are inappropriate for evanescent modes and inaccurate for grazing waves. For parabolic wave equations, Milinazzo et al. [63] proposed the following modified approximation with a change of variable to rotate the branch cut of the square root by some angle $\phi$, which leads to

$$
f_{2 N+1}^{\phi}(X)=e^{\imath \phi / 2} f_{2 N+1}^{\text {Padé }}\left(e^{-\imath \phi}(1+X)-1\right)=e^{\imath \phi / 2}\left[1+\frac{2}{M} \sum_{i=1}^{N} c_{i}\left(1-\frac{e^{\imath \phi}\left(c_{i}+1\right)}{\left(e^{\imath \phi} c_{i}+1\right)+X}\right)\right] .
$$

The obtained approximation highly improves the accuracy of the HABC for evanescent modes [8], while it slightly increases the reflection of traveling modes. The effect is more important as the rotating angle $\phi$ grows.

More general approximations of the DtN operator have been proposed (see e.g. [41, 44-46, 49, 59, 69]), leading to absorbing boundary conditions possibly more accurate thanks to a larger number of tuning parameters [41, 44, 69]. In particular, Higdon-type HABCs [49] are exact for waves propagating with any angle of incidence of a given set $\left\{\theta_{m}\right\}_{m=1 \ldots M}$, but they are still inefficient for evanescent waves. The complete radiation boundary conditions (CRBCs) can deal with both propagative and evanescent modes. In the time-harmonic context, these boundary conditions are equivalent to the general boundary condition

$$
\left[\prod_{m=1}^{M}\left(\partial_{x}-\imath \kappa \alpha_{m}\right)\right] u=0
$$


which corresponds to the rational approximation of the square root $f_{M}(X)$ provided by the sequence

$$
\begin{aligned}
f_{m}(X) & =\alpha_{m}+\frac{X+1-\alpha_{m}^{2}}{\alpha_{m}+f_{m-1}(X)}, \quad \text { for } m=2 \ldots M, \\
f_{1}(X) & =\alpha_{1},
\end{aligned}
$$

where $\left\{\alpha_{m}\right\}_{m=1 \ldots M}$ are complex coefficients. The real and imaginary parts of these coefficients attenuate respectively propagative and evanescent parts of waves. We have the following coefficients (see A for further details):

- Padé-type HABC without rotation of the branch cut [31, 32]: $\alpha_{m}=1$;

- Padé-type HABC with $\phi$-rotation of the branch cut [53]: $\alpha_{m}=e^{\imath \phi / 2}$;

- Higdon-type $\operatorname{HABC}[40,43,49]: \alpha_{m}=\cos \theta_{m}$, with $\theta_{m} \in[0, \pi / 2[$;

- CRBC [44]: $\alpha_{m}=\cos \theta_{m}-\sigma_{m} /(\imath \kappa)$, with $\theta_{m} \in\left[0, \pi / 2\left[\right.\right.$ and $\sigma_{m}>0$.

In an alternative version of the CRBC, the coefficients are separated into a set of purely real parameters and a set of purely imaginary parameters [41, 45]. The convergence of that CRBC has been analysed for waveguides in [41]. To the best of our knowledge, the convergence of HABCs has not yet been addressed for high-frequency scattering problems.

In this work, we limit ourself to the $\phi$-rotated Padé-type approximation written as a sum of prime fractions (3). We recognize that this approximation is a particular case of the CRBC with $\theta_{m}=\phi / 2$ and $\sigma_{m}=\kappa \sin (\phi / 2)$ for $m=1 \ldots M$. Although the $\phi$-rotated Padé-type HABCs are potentially less accurate than fully-optimized CRBCs, they are of practical interest since only two parameters must be selected $(\phi$ and $M)$. The parameter selection is analyzed with numerical results in section 3.3. Let us note that these conditions could be easily extended to more general approximations by considering $\left\{c_{i}\right\}_{i=1 \ldots N}$ as complex parameters to be tuned.

\subsection{Padé-type high-order absorbing boundary condition}

Using the approximation (3) in the pseudo-differential operator (1) leads to the approximate boundary condition

$$
\left.\partial_{x} u\right|_{\Gamma}=\left.\mathcal{R} u\right|_{\Gamma}, \quad \text { on } \Gamma,
$$

where $\mathcal{R}$ is the pseudo-differential operator defined as

$$
\mathcal{R} \equiv \imath \kappa e^{\imath \phi / 2}\left[1+\frac{2}{M} \sum_{i=1}^{N} c_{i}\left(1-\frac{e^{\imath \phi}\left(c_{i}+1\right)}{\left(e^{\imath \phi} c_{i}+1\right)+\Delta_{\Gamma} / \kappa^{2}}\right)\right] .
$$

The field $u(x, y, z)$ denotes the solution of the truncated problem defined on $\Omega$, with the approximate condition on $\Gamma$. This solution is an approximation of the exact free-space solution $v(x, y, z)$, with spurious reflections generated at the boundary. Following a strategy used by Lindman [58] and next by Collino [24, 25], high-order absorbing boundary conditions can be written with differential equations by introducing auxiliary surface fields and additional equations on the boundary. Here, we define $N$ auxiliary fields $\varphi_{i}$, with $i=1 \ldots N$, governed by

$$
\mathcal{N}_{i} \varphi_{i}=u, \quad \text { for } i=1 \ldots N, \quad \text { on } \Gamma,
$$

where $\mathcal{N}_{i}$ is the differential operator given by

$$
\mathcal{N}_{i} \equiv-\frac{\left(e^{\imath \phi} c_{i}+1\right)+\Delta_{\Gamma} / \kappa^{2}}{e^{\imath \phi}\left(c_{i}+1\right)} .
$$

Using these auxiliary fields, the boundary condition (4) can be rewritten as

$$
\left.\partial_{x} u\right|_{\Gamma}=\mathcal{L}\left(\left.u\right|_{\Gamma}, \varphi_{1}, \ldots, \varphi_{N}\right), \quad \text { on } \Gamma,
$$


where $\mathcal{L}$ is the linear algebraic operator defined as

$$
\mathcal{L}\left(g, g_{1}, \ldots, g_{N}\right) \equiv \imath \kappa e^{\imath \phi / 2}\left[g+\frac{2}{M} \sum_{i=1}^{N} c_{i}\left(g+g_{i}\right)\right] .
$$

This boundary condition and the auxiliary equations (5) are local equations. Writing explicitly these relations finally gives the $\mathrm{HABC}$

$$
\left.\partial_{x} u\right|_{\Gamma}=\imath \kappa e^{\imath \phi / 2}\left[\left.u\right|_{\Gamma}+\frac{2}{M} \sum_{i=1}^{N} c_{i}\left(\left.u\right|_{\Gamma}+\varphi_{i}\right)\right], \quad \text { on } \Gamma,
$$

and the auxiliary equations

$$
\Delta_{\Gamma} \varphi_{i}+\kappa^{2}\left(\left(e^{\imath \phi} c_{i}+1\right) \varphi_{i}+\left.e^{\imath \phi}\left(c_{i}+1\right) u\right|_{\Gamma}\right)=0, \quad \text { for } i=1 \ldots N, \quad \text { on } \Gamma .
$$

When using this boundary condition, two parameters must be chosen: the angle $\phi$ and the number of auxiliary fields $N$, which are related to the approximation of the square root.

\section{Exact treatment for settings with right angles}

When using the HABC on the faces of polygonal or polyhedral domains, the corners and the edges of these domains require a specific treatment. To illustrate this, let us consider the two-dimensional Helmholtz problem defined on the quarter space $\Omega=\left\{(x, y) \in \mathbb{R}^{2}: x<0, y<0\right\}$. On both edges of $\Omega$, we prescribe a $\mathrm{HABC}$, i.e.

$$
\begin{array}{ll}
\left.\partial_{x} u\right|_{\Gamma^{x}}=\imath \kappa e^{\imath \phi / 2}\left[\left.u\right|_{\Gamma^{x}}+\frac{2}{M} \sum_{i=1}^{N} c_{i}\left(\left.u\right|_{\Gamma^{x}}+\varphi_{i}^{x}\right)\right], & \text { on } \Gamma^{x}, \\
\left.\partial_{y} u\right|_{\Gamma^{y}}=\imath \kappa e^{\imath \phi / 2}\left[\left.u\right|_{\Gamma^{y}}+\frac{2}{M} \sum_{j=1}^{N} c_{j}\left(\left.u\right|_{\Gamma^{y}}+\varphi_{j}^{y}\right)\right], & \text { on } \Gamma^{y},
\end{array}
$$

with auxiliary fields governed by

$$
\begin{aligned}
& \partial_{y y} \varphi_{i}^{x}+\kappa^{2}\left(\left(e^{\imath \phi} c_{i}+1\right) \varphi_{i}^{x}+\left.e^{\imath \phi}\left(c_{i}+1\right) u\right|_{\Gamma^{x}}\right)=0, \quad \text { for } i=1 \ldots N, \quad \text { on } \Gamma^{x}, \\
& \partial_{x x} \varphi_{j}^{y}+\kappa^{2}\left(\left(e^{\imath \phi} c_{j}+1\right) \varphi_{j}^{y}+\left.e^{\imath \phi}\left(c_{j}+1\right) u\right|_{\Gamma^{y}}\right)=0, \quad \text { for } j=1 \ldots N, \quad \text { on } \Gamma^{y} \text {, }
\end{aligned}
$$

where $\Gamma^{x}=\left\{(x, y) \in \mathbb{R}^{2}: x=0, y<0\right\}$ and $\Gamma^{y}=\left\{(x, y) \in \mathbb{R}^{2}: x<0, y=0\right\}$. From a mathematical point of view, boundary conditions must be prescribed on these auxiliary fields at the boundary of each edge (i.e. at the corner $\left.P^{x y}=(0,0)\right)$ because of the second-order spatial derivative arising in equations (10)-(11). From a modeling point of view, these conditions provide additional information about the exterior problem. Indeed, if we seek the solution of the free-space problem, the corner treatment should accurately represents the outward propagation of waves at the corner. A similar reasoning can be made in three dimensions.

In this section, we present and analyze a strategy to deal with the corners and the edges of rectangular and cuboidal domains, which have only right angles. The strategy relies on additional relations that give the missing boundary conditions, and supplementary auxiliary fields defined on the corners and the edges. The approach is explained in section 3.1 in the two-dimensional case, and extended in section 3.2 for the three-dimensional case. Numerical results are proposed in section 3.3 to assert the efficiency of the corner treatment and to study the parameters selection.

\subsection{Compatibility relations in two dimensions}

Let us remind that we seek an approximate solution of the free-space problem

$$
\Delta v+\kappa^{2} v=s, \quad \text { in } \mathbb{R}^{2},
$$


where $v(x, y)$ is the exact free-space solution verifying the Sommerfeld's condition, the source term $f(x, y)$ has a compact support on the quarter space $\Omega$, and the wavenumber $\kappa(x, y)$ is constant in $\mathbb{R}^{2} \backslash \Omega$. The exterior region $\mathbb{R}^{2} \backslash \Omega$ contains the two half spaces $\Omega^{x}=\left\{(x, y) \in \mathbb{R}^{2}: x>0\right\}$ and $\Omega^{y}=\left\{(x, y) \in \mathbb{R}^{2}\right.$ : $y>0\}$, which overlap on the exterior quarter space $\Omega^{x y}=\left\{(x, y) \in \mathbb{R}^{2}: x>0, y>0\right\}$. Following the procedure detailed in the previous section, solving the exterior problems defined on the half spaces $\Omega^{x}$ and $\Omega^{y}$ leads to the exact relations

$$
\begin{array}{ll}
\left.\partial_{x} v\right|_{x=0}=\left.\mathcal{B}^{x} v\right|_{x=0}, & \text { for } y \in \mathbb{R}, \\
\left.\partial_{y} v\right|_{y=0}=\left.\mathcal{B}^{y} v\right|_{y=0}, & \text { for } x \in \mathbb{R},
\end{array}
$$

with the DtN operators $\mathcal{B}^{x}$ and $\mathcal{B}^{y}$ formally defined as

$$
\begin{aligned}
\mathcal{B}^{x} & \equiv \imath \kappa \sqrt{1+\partial_{y y} / k^{2}}, \\
\mathcal{B}^{y} & \equiv \imath \kappa \sqrt{1+\partial_{x x} / k^{2}} .
\end{aligned}
$$

In the truncated problem defined on $\Omega$, these two operators are localized using a Padé-type approximation of the square root, and the restriction of the obtained approximate boundary conditions on $\Gamma^{x}$ and $\Gamma^{y}$ are used. It leads to equations (8) and (9) and the approximate solution $u(x, y)$.

In order to derive a suited corner treatment, let us consider the solutions of particular exterior halfspace problems defined on $\Omega^{x}$ and $\Omega^{y}$, which verify the HABC at the interface of the respective interior domain. The exterior half-space problem on $\Omega^{x}$ reads

$$
\left\{\begin{aligned}
\Delta \tilde{u}^{x}+k^{2} \tilde{u}^{x} & =0, & & \text { in } \Omega^{x}, \\
\tilde{u}^{x} & =\bar{u}^{x}(y), & & \text { for } x=0, y \in \mathbb{R}, \\
\partial_{x} \tilde{u}^{x} & =\mathcal{L}\left(\tilde{u}^{x}, \tilde{\varphi}_{1}^{x}, \ldots, \tilde{\varphi}_{N}^{x}\right), & & \text { for } x=0, y \in \mathbb{R},
\end{aligned}\right.
$$

with auxiliary fields defined as

$$
\mathcal{N}_{i}^{x} \tilde{\varphi}_{i}^{x} \equiv \tilde{u}^{x}, \quad \text { for } i=1 \ldots N, \quad \text { in } \Omega^{x},
$$

where the differential operator $\mathcal{N}_{i}^{x}$ is

$$
\mathcal{N}_{i}^{x} \equiv-\frac{\left(e^{\imath \phi} c_{i}+1\right)+\partial_{y y} / \kappa^{2}}{e^{\imath \phi}\left(c_{i}+1\right)} .
$$

Both the main field $\tilde{u}^{x}$ and the auxiliary fields $\tilde{\varphi}_{i}^{x}$, denoted with a tilde , are defined on the exterior half space $\Omega^{x}$. Since $\mathcal{N}_{i}^{x}$ is a dissipative Helmholtz-type operator, it can be inverted. Therefore, the auxiliary fields $\tilde{\varphi}_{i}^{x}$ are defined uniquely through (14), and we can write

$$
\tilde{\varphi}_{i}^{x}=\left(\mathcal{N}_{i}^{x}\right)^{-1} \tilde{u}^{x}, \quad \text { for } i=1 \ldots N, \quad \text { in } \Omega^{x} .
$$

Similarly, we introduce the exterior half-space problem for the unknown $\tilde{u}^{y}$ and the auxiliary fields $\tilde{\varphi}_{j}^{y}=\left(\mathcal{N}_{j}^{y}\right)^{-1} \tilde{u}^{y}$, for $j=1 \ldots N$, defined on $\Omega^{y}$. The operator $\mathcal{N}_{j}^{y}$ is defined by equation (15) with $\partial_{y y}$ instead of $\partial_{x x}$.

Because of the spatial derivatives in equations (10)-(11), we seek for supplementary boundary conditions giving the traces $\left.\partial_{y} \varphi_{i}^{x}\right|_{P^{x y}}$ and $\left.\partial_{x} \varphi_{j}^{y}\right|_{P^{x y}}$ at the corner. To do this, we first enforce the approximate half-space fields $\tilde{u}^{x}$ and $\tilde{u}^{y}$ to be equal in the exterior quarter space, i.e. $\tilde{u}^{x}=\tilde{u}^{y}=\tilde{u}$ in $\Omega^{x y}$. Because we have a right angle between the interfaces, the normal derivative of one face is the tangential derivative of the other face up to a sign. Therefore, since $\mathcal{L}$ is a linear operator, by applying $\left(\mathcal{N}_{j}^{y}\right)^{-1}$ on the last equation of $(13)$ and $\left(\mathcal{N}_{i}^{x}\right)^{-1}$ on the corresponding equation for $\partial_{y} \tilde{u}^{y}$, we obtain

$$
\begin{aligned}
& \partial_{x} \tilde{\varphi}_{j}^{y}=\mathcal{L}\left(\tilde{\varphi}_{j}^{y}, \tilde{\varphi}_{1 j}^{y}, \ldots, \tilde{\varphi}_{N j}^{y}\right), \quad \text { for } j=1 \ldots N, \quad \text { for } x=0, y>0, \\
& \partial_{y} \tilde{\varphi}_{i}^{x}=\mathcal{L}\left(\tilde{\varphi}_{i}^{x}, \tilde{\varphi}_{i 1}^{x}, \ldots, \tilde{\varphi}_{i N}^{x}\right), \quad \text { for } i=1 \ldots N, \quad \text { for } x>0, y=0,
\end{aligned}
$$

where we have introduced new fields

$$
\begin{array}{ll}
\tilde{\varphi}_{i j}^{y}=\left(\mathcal{N}_{j}^{y}\right)^{-1} \tilde{\varphi}_{i}^{x}, & \text { for } i, j=1 \ldots N, \quad \text { in } \Omega^{x y}, \\
\tilde{\varphi}_{i j}^{x}=\left(\mathcal{N}_{i}^{x}\right)^{-1} \tilde{\varphi}_{j}^{y}, & \text { for } i, j=1 \ldots N, \quad \text { in } \Omega^{x y} .
\end{array}
$$


The restriction of equations (16) and (17) at the corner gives the missing boundary conditions. However, they require the computation of the auxiliary fields $\tilde{\varphi}_{i j}^{x}$ and $\tilde{\varphi}_{i j}^{y}$ only at the corner, while the governing equations (18)-(19) involve spatial derivatives of these fields. To overcome this problem, we first note that $\tilde{\varphi}_{i j}^{x}=\tilde{\varphi}_{i j}^{y}$, for $i, j=1 \ldots N$. Indeed, since $\mathcal{N}_{i}^{x}$ and $\mathcal{N}_{j}^{y}$ commute $\left(i . e . \mathcal{N}_{i}^{x} \mathcal{N}_{j}^{y}=\mathcal{N}_{j}^{y} \mathcal{N}_{i}^{x}\right)$ and can be inverted, we have

$$
\tilde{\varphi}_{i j}^{x y} \equiv \tilde{\varphi}_{i j}^{x}=\left(\mathcal{N}_{i}^{x}\right)^{-1}\left(\mathcal{N}_{j}^{y}\right)^{-1} \tilde{u}=\left(\mathcal{N}_{j}^{y}\right)^{-1}\left(\mathcal{N}_{i}^{x}\right)^{-1} \tilde{u}=\tilde{\varphi}_{i j}^{y}, \quad \text { for } i, j=1 \ldots N, \quad \text { in } \Omega^{x y} .
$$

We can then explicitly write equations (18) and (19) as

$$
\begin{array}{lll}
\partial_{y y} \tilde{\varphi}_{i j}^{x y}+\kappa^{2}\left(\left(e^{\imath \phi} c_{i}+1\right) \tilde{\varphi}_{i j}^{x y}+e^{\imath \phi}\left(c_{i}+1\right) \tilde{\varphi}_{j}^{y}\right)=0, & \text { for } i, j=1 \ldots N, & \text { in } \Omega^{x y}, \\
\partial_{x x} \tilde{\varphi}_{i j}^{x y}+\kappa^{2}\left(\left(e^{\imath \phi} c_{j}+1\right) \tilde{\varphi}_{i j}^{x y}+e^{\imath \phi}\left(c_{j}+1\right) \tilde{\varphi}_{i}^{x}\right)=0, & \text { for } i, j=1 \ldots N, & \text { in } \Omega^{x y} .
\end{array}
$$

Since the Helmholtz operator commutes with $\mathcal{N}_{j}^{y}$ and $\mathcal{N}_{i}^{x}$, the auxiliary fields also verify the Helmholtz equation,

$$
\Delta \tilde{\varphi}_{i j}^{x y}+\kappa^{2} \tilde{\varphi}_{i j}^{x y}=0, \quad \text { for } i, j=1 \ldots N, \quad \text { in } \Omega^{x y} .
$$

Canceling the spatial derivatives by combining the three previous equations gives the algebraic relations

$$
\left(e^{\imath \phi} c_{i}+e^{\imath \phi} c_{j}+1\right) \tilde{\varphi}_{i j}^{x y}+e^{\imath \phi}\left(c_{i}+1\right) \tilde{\varphi}_{j}^{y}+e^{\imath \phi}\left(c_{j}+1\right) \tilde{\varphi}_{i}^{x}=0, \quad \text { for } i, j=1 \ldots N, \quad \text { in } \Omega^{x y} .
$$

Finally, taking the restriction of equations (16), (17) and (20) at the corner $P^{x y}$ gives the missing boundary conditions for the truncated problem,

$$
\begin{aligned}
& \left.\partial_{x} \varphi_{j}^{y}\right|_{P^{x y}}=\imath \kappa e^{\imath \phi / 2}\left[\left.\varphi_{j}^{y}\right|_{P^{x y}}+\frac{2}{M} \sum_{i=1}^{N} c_{i}\left(\left.\varphi_{j}^{y}\right|_{P^{x y}}+\varphi_{i j}^{x y}\right)\right], \quad \text { for } j=1 \ldots N, \quad \text { at } P^{x y} \\
& \left.\partial_{y} \varphi_{i}^{x}\right|_{P^{x y}}=\imath \kappa e^{\imath \phi / 2}\left[\left.\varphi_{i}^{x}\right|_{P^{x y}}+\frac{2}{M} \sum_{j=1}^{N} c_{j}\left(\left.\varphi_{i}^{x}\right|_{P^{x y}}+\varphi_{i j}^{x y}\right)\right], \quad \text { for } i=1 \ldots N, \quad \text { at } P^{x y},
\end{aligned}
$$

with $N^{2}$ auxiliary fields defined at the corner and given by

$$
\varphi_{i j}^{x y}=-\frac{\left.\left(c_{j}+1\right) \varphi_{i}^{x}\right|_{P^{x y}}+\left.\left(c_{i}+1\right) \varphi_{j}^{y}\right|_{P^{x y}}}{c_{i}+c_{j}+e^{-\imath \phi}}, \quad \text { for } i, j=1 \ldots N, \quad \text { at } P^{x y} .
$$

In a nutshell, the corner treatment consists in applying the HABC on the $2 N$ auxiliary fields belonging to the edges, and they are coupled through $N^{2}$ supplementary auxiliary fields defined only at the corner.

When solving these equations with a finite element scheme, we straightforwardly adapt the bilinear form of the Helmholtz equation. The HABC (equations (8)-(9)), the auxiliary equations (equations (10)(11)) and the compatibility relations (equations (21)-(22)) are directly incorporated in the variational formulation of the problem. The variational formulation is provided in B.

\subsection{Compatibility relations in three dimensions}

Our approach can be applied in three dimensions. When using the HABC on all the faces of a cuboidal domain to represent wave propagation in the free space $\mathbb{R}^{3}$, additional relations must be prescribed at the edges and the corners of the cuboid. To state the equations, we consider the three-dimensional version of the Helmholtz problem (12) and a truncated domain that is the eighth of space $\Omega=\left\{(x, y, z) \in \mathbb{R}^{3}\right.$ : $x<0, y<0, z<0\}$. The faces of $\Omega$ belonging to the planes $x=0, y=0$ and $z=0$ are denoted $\Gamma^{x}, \Gamma^{y}$ and $\Gamma^{z}$, respectively. The edges are given by $\Upsilon^{x y}=\overline{\Gamma^{x}} \cap \overline{\Gamma^{y}}, \Upsilon^{x z}=\overline{\Gamma^{x}} \cap \overline{\Gamma^{z}}$ and $\Upsilon^{y z}=\overline{\Gamma^{y}} \cap \overline{\Gamma^{z}}$. The corner is defined as $P^{x y z}=\overline{\Gamma^{x}} \cap \overline{\Gamma^{y}} \cap \overline{\Gamma^{z}}$.

On the faces $\Gamma^{x}, \Gamma^{y}$ and $\Gamma^{z}$, the boundary conditions for the approximate solution $u(x, y, z)$ read

$$
\begin{array}{lll}
\left.\partial_{x} u\right|_{\Gamma_{x}}=\mathcal{L}\left(\left.u\right|_{\Gamma_{x}}, \varphi_{1}^{x}, \ldots, \varphi_{N}^{x}\right), & & \text { on } \Gamma^{x}, \\
\left.\partial_{y} u\right|_{\Gamma_{y}}=\mathcal{L}\left(\left.u\right|_{\Gamma_{y}}, \varphi_{1}^{y}, \ldots, \varphi_{N}^{y}\right), & & \text { on } \Gamma^{y}, \\
\left.\partial_{z} u\right|_{\Gamma_{z}}=\mathcal{L}\left(\left.u\right|_{\Gamma_{z}}, \varphi_{1}^{z}, \ldots, \varphi_{N}^{z}\right), & & \text { on } \Gamma^{z},
\end{array}
$$


with $N$ auxiliary fields defined on each face and governed by

$$
\begin{aligned}
\left(\partial_{y y}+\partial_{z z}\right) \varphi_{i}^{x}+\kappa^{2} e^{\imath \phi}\left(\left(c_{i}+e^{-\imath \phi}\right) \varphi_{i}^{x}+\left.\left(c_{i}+1\right) u\right|_{\Gamma^{x}}\right) & =0, & \forall i, & \text { on } \Gamma^{x}, \\
\left(\partial_{x x}+\partial_{z z}\right) \varphi_{j}^{y}+\kappa^{2} e^{\imath \phi}\left(\left(c_{j}+e^{-\imath \phi}\right) \varphi_{j}^{y}+\left.\left(c_{j}+1\right) u\right|_{\Gamma^{y}}\right) & =0, & \forall j, & \text { on } \Gamma^{y}, \\
\left(\partial_{x x}+\partial_{y y}\right) \varphi_{k}^{z}+\kappa^{2} e^{\imath \phi}\left(\left(c_{k}+e^{-\imath \phi}\right) \varphi_{k}^{z}+\left.\left(c_{k}+1\right) u\right|_{\Gamma^{z}}\right) & =0, & \forall k, & \text { on } \Gamma^{z} .
\end{aligned}
$$

For the sake of conciseness, we do no write that the values of the indices $i, j$ and $k$ are between 1 and $N$. Because of the spatial derivative in their governing equations, the auxiliary fields defined on the faces require boundary conditions on the edges. By extending the approach of the previous section, we obtain

$$
\begin{aligned}
& \left.\partial_{y} \varphi_{i}^{x}\right|_{\Upsilon^{x y}}=\mathcal{L}\left(\left.\varphi_{i}^{x}\right|_{\Upsilon^{x y}}, \varphi_{i 1}^{x y}, \ldots, \varphi_{i N}^{x y}\right), \quad \forall i, \quad \text { on } \Upsilon^{x y} \\
& \left.\partial_{z} \varphi_{i}^{x}\right|_{\Upsilon^{x z}}=\mathcal{L}\left(\left.\varphi_{i}^{x}\right|_{\Upsilon^{x z}}, \varphi_{i 1}^{x z}, \ldots, \varphi_{i N}^{x z}\right), \quad \forall i, \quad \text { on } \Upsilon^{x z}, \\
& \left.\partial_{x} \varphi_{j}^{y}\right|_{\Upsilon^{x y}}=\mathcal{L}\left(\left.\varphi_{j}^{y}\right|_{\Upsilon^{x y}}, \varphi_{1 j}^{x y}, \ldots, \varphi_{N j}^{x y}\right), \quad \forall j, \quad \text { on } \Upsilon^{x y}, \\
& \left.\partial_{z} \varphi_{j}^{y}\right|_{\Upsilon y z}=\mathcal{L}\left(\left.\varphi_{j}^{y}\right|_{\Upsilon y z}, \varphi_{j 1}^{y z}, \ldots, \varphi_{j N}^{y z}\right), \quad \forall j, \quad \text { on } \Upsilon^{y z} \text {, } \\
& \left.\partial_{x} \varphi_{k}^{z}\right|_{\Upsilon^{x z}}=\mathcal{L}\left(\left.\varphi_{k}^{z}\right|_{\Upsilon^{x z}}, \varphi_{1 k}^{x z}, \ldots, \varphi_{N k}^{x z}\right), \quad \forall k, \quad \text { on } \Upsilon^{x z} \text {, } \\
& \left.\partial_{y} \varphi_{k}^{z}\right|_{\Upsilon y z}=\mathcal{L}\left(\left.\varphi_{k}^{z}\right|_{\Upsilon^{y z}}, \varphi_{1 k}^{y z}, \ldots, \varphi_{N k}^{y z}\right), \quad \forall k, \quad \text { on } \Upsilon^{y z},
\end{aligned}
$$

with $N^{2}$ auxiliary fields defined on each edge and governed by

$$
\begin{aligned}
& \partial_{z z} \varphi_{i j}^{x y}+\kappa^{2} e^{\imath \phi}\left(\left(c_{i}+c_{j}+e^{-\imath \phi}\right) \varphi_{i j}^{x y}+\left(c_{i}+1\right) \varphi_{j}^{x}\left|\Upsilon^{x y}+\left(c_{j}+1\right) \varphi_{i}^{x}\right| \Upsilon^{x y}\right)=0, \quad \forall i, j, \quad \text { on } \Upsilon^{x y}, \\
& \partial_{y y} \varphi_{i k}^{x z}+\kappa^{2} e^{\imath \phi}\left(\left(c_{i}+c_{k}+e^{-\imath \phi}\right) \varphi_{i k}^{x z}+\left(c_{i}+1\right) \varphi_{k}^{x}\left|\Upsilon^{x z}+\left(c_{k}+1\right) \varphi_{i}^{x}\right| \Upsilon^{x z}\right)=0, \quad \forall i, k, \quad \text { on } \Upsilon^{x z}, \\
& \partial_{x x} \varphi_{j k}^{y z}+\kappa^{2} e^{\imath \phi}\left(\left(c_{j}+c_{k}+e^{-\imath \phi}\right) \varphi_{j k}^{y z}+\left.\left(c_{j}+1\right) \varphi_{k}^{x}\right|_{\Upsilon y z}+\left.\left(c_{k}+1\right) \varphi_{j}^{x}\right|_{\Upsilon y z}\right)=0, \quad \forall j, k, \quad \text { on } \Upsilon^{y z} .
\end{aligned}
$$

Again, boundary conditions must be prescribed at the corner on the auxiliary fields belonging to the edges because of the spatial derivative in the governing equations. We obtain the boundary conditions

$$
\begin{aligned}
& \left.\partial_{z} \varphi_{i j}^{x y}\right|_{P^{x y z}}=\mathcal{L}\left(\left.\varphi_{i j}^{x y}\right|_{P^{x y z}}, \varphi_{i j 1}^{x y z}, \ldots, \varphi_{i j N}^{x y z}\right), \quad \forall i, j, \quad \text { on } P^{x y z}, \\
& \left.\partial_{y} \varphi_{i k}^{x z}\right|_{P^{x y z}}=\mathcal{L}\left(\left.\varphi_{i k}^{x z}\right|_{P^{x y z}}, \varphi_{i 1 k}^{x y z}, \ldots, \varphi_{i N k}^{x y z}\right), \quad \forall i, k, \quad \text { on } P^{x y z}, \\
& \left.\partial_{x} \varphi_{j k}^{y z}\right|_{P^{x y z}}=\mathcal{L}\left(\left.\varphi_{j k}^{y z}\right|_{P^{x y z}}, \varphi_{1 j k}^{x y z}, \ldots, \varphi_{N j k}^{x y z}\right), \quad \forall j, k, \quad \text { on } P^{x y z},
\end{aligned}
$$

with $N^{3}$ auxiliary fields given by

$$
\varphi_{i j k}^{x y z}=-\frac{\left.\left(c_{i}+1\right) \varphi_{j k}^{y z}\right|_{P^{x y z}}+\left.\left(c_{j}+1\right) \varphi_{i k}^{x z}\right|_{P^{x y z}}+\left.\left(c_{k}+1\right) \varphi_{i j}^{x y}\right|_{P^{x y z}}}{c_{i}+c_{j}+c_{k}+e^{-\imath \phi}}, \quad \forall i, j, k, \quad \text { on } P^{x y z} .
$$

Therefore, for using this approach with a cuboidal domain, we must define two-dimensional auxiliary fields on the faces ( $N$ per face), one-dimensional auxiliary fields on the edges $\left(N^{2}\right.$ per edge) and additional scalar unknowns at the corners $\left(N^{3}\right.$ per corner). Auxiliary differential equations similar to the twodimensional and one-dimensional versions of the Helmholtz equation must be solved on the faces and the edges, respectively.

\subsection{Numerical verification and parameter analysis}

In this section, the accuracy of the $\mathrm{HABC}$ with the compatibility relations at corners and edges is asserted by considering two reference benchmarks: the scattering of a plane wave by a circular cylinder and by a sphere. The influence of the parameters $\phi$ and $N$ is analyzed in two dimensions. The numerical results have been obtained with the mesh generator Gmsh [35] and the finite element solver GetDP [29].

\section{Numerical verification in two dimensions}

The scattering of the plane wave solution $u^{\text {inc }}(\vec{x})=e^{\imath \kappa x}$ with the propagation direction $\hat{\vec{\kappa}}=[1,0]$ by the sound-hard circular cylinder of radius $R$ centered at the origin generates the scattered field

$$
u^{\mathrm{ref}}(r, \theta)=-\sum_{m=0}^{\infty} \epsilon_{m} \imath^{m} \frac{J_{m}{ }^{\prime}(\kappa R)}{H_{m}^{(1)^{\prime}}(\kappa R)} H_{m}^{(1)}(\kappa r) \cos (m \theta), \quad r \geq R,
$$


(a) Reference solution

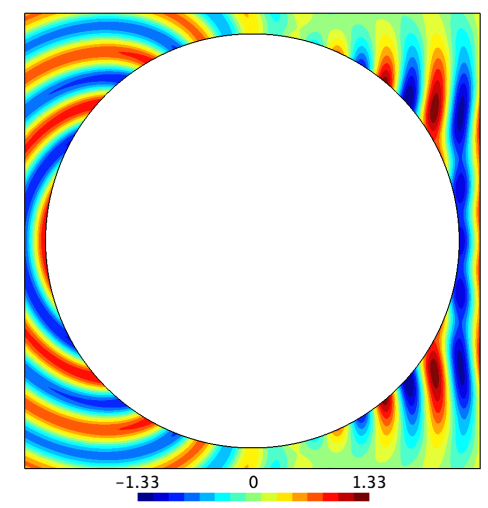

(b) Error with $\phi=0$

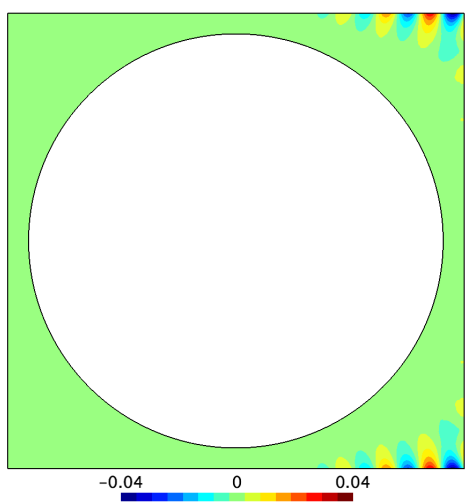

(c) Error with $\phi=\pi / 3$

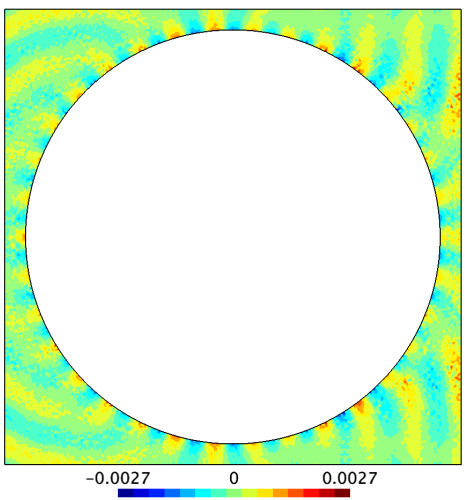

Figure 1: Scattering by a sound-hard circular cylinder: real part of the reference solution $\Re e\left\{u^{\text {ref }}\right\}$ (Figure 1a) and real part of the error $\Re e\left\{u^{\text {num }}-u^{\text {ref }}\right\}$ when using the HABC with the exact corner treatment, $N=4$ and either $\phi=0$ (Figure 1b) or $\phi=\pi / 3$ (Figure 1c). The mesh is made of 7,156 elements and 14,917 second-order nodes, which corresponds to $n_{\lambda} \approx 10$.

(a) HABC with $\phi=0$

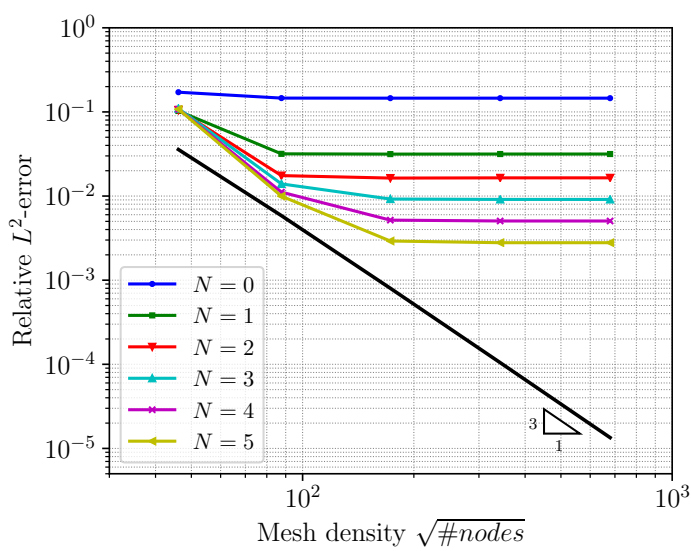

(b) HABC with $\phi=\pi / 3$

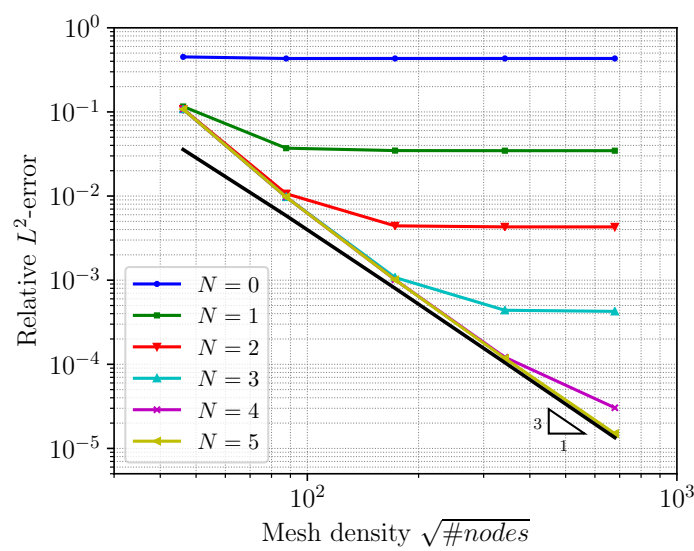

Figure 2: Scattering by a sound-hard circular cylinder: convergence of the numerical solution. The relative $L^{2}$-error takes into account both modeling errors due to the HABC and numerical errors due to the scheme. In both figures, the black line corresponds to the relative projection $L^{2}$-error.

where $(r, \theta)$ are the polar coordinates, $J_{m}$ is the $m^{\text {th }}$-order Bessel's function, $H_{m}^{(1)}$ is the $m^{\text {th }}$-order firstkind Hankel function, and $\epsilon_{m}$ is the Neumann function which is equal to 1 for $m=0$ and 2 otherwise.

We consider a two-dimensional setting where a disk of radius $R=1$ is placed in the middle of the squared domain $[-1.1,1.1]^{2}$. The Neumann boundary condition $\left.\partial_{n} u\right|_{\partial \Omega^{\text {disk }}}=-\left.\partial_{n} u^{\text {inc }}\right|_{\partial \Omega^{\text {disk }}}$ is prescribed on the boundary of the disk, HABCs are set on all the edges of the square, and the compatibility relations are used at the corners. The scattered field solution is computed for the wavenumber $\kappa=25$ on meshes made of second-order curvilinear triangular elements with second-order nodal basis functions. The real part of the reference solution is shown in Figure 1a for this setting.

Figures $1 \mathrm{~b}$ and $1 \mathrm{c}$ show the error on the numerical solutions computed with $N=4$ auxiliary fields and either $\phi=0$ or $\phi=\pi / 3$, respectively. In the first case, the error is dominated by surface waves propagating along the upper and lower artificial boundaries. These spurious waves are canceled when rotating the branch cut of by a $\pi / 3$ angle in the Padé approximation, and the standard dispersive numerical error dominates the global error.

To quantify the accuracy of the HABC with the corner compatibility relations, we study the global error between the numerical solution $u^{\text {num }}$ and the reference solution $u^{\text {ref }}$ on the computational domain $\Omega$. This error takes into account both modeling errors due to the approximate boundary treatment 
and numerical errors due to the numerical approximations. If the boundary treatment is sufficiently accurate, the global error will be dominated by numerical errors, and the third-order convergence rate of the numerical scheme should be recovered.

In Figure 2, we show the relative $L^{2}$-error as a function of the mesh density for five meshes in different settings: the number of auxiliary fields $N=0,1,2,3,4$ and 5 and the angles $\phi=0$ and $\pi / 3$. The ratio of the characteristic size of a mesh cell to the wavelength is $n_{\lambda} \approx 2.5,5,10,20,40$ for the five meshes. The relative $L^{2}$-error is defined as

$$
\frac{\left\|u^{\mathrm{num}}-u^{\mathrm{ref}}\right\|_{L^{2}(\Omega)}}{\left\|u^{\mathrm{ref}}\right\|_{L^{2}(\Omega)}},
$$

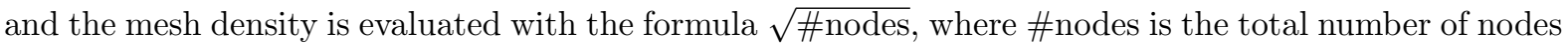
in the mesh (i.e. with 6 nodes per triangle). In addition, we also show the relative $L^{2}$-error between the reference solution and its $L^{2}$-projection onto the finite element space,

$$
\frac{\left\|\mathcal{P} u^{\mathrm{ref}}-u^{\mathrm{ref}}\right\|_{L^{2}(\Omega)}}{\left\|u^{\mathrm{ref}}\right\|_{L^{2}(\Omega)}}
$$

By Céa's lemma, this error corresponds to the best numerical solution that can be done on each mesh, whatever the boundary treatment.

For the coarsest meshes, the smallest numerical error is reached with a small number of auxiliary fields $N$, both for $\phi=0$ and $\phi=\pi / 3$, but this error is significantly higher than the projection error. When refining the mesh, the numerical error decreases until a plateau in all the cases. This plateau corresponds to the modeling error due to the approximate treatment at the boundary. Increasing $N$ decreases the level of the plateau, and taking $\phi=\pi / 3$ instead of $\phi=0$ accelerates this decrease. For $N=5$ and $\phi=\pi / 3$, we observe that the numerical error reaches the projection error for the finest meshes. The numerical solutions obtained with the HABC then are very close to the best approximations possible with these meshes, and the modeling error is negligible.

\section{Parameter analysis in two dimensions}

To understand the role of the angle $\phi$, and then to guide the parameter selection, we analyse the error for each mode of the solution. The incident wave can be decomposed into the modes $u_{m}^{\mathrm{inc}}(r, \theta)=J_{m}(\kappa r) e^{\imath m \theta}$, where $m \in \mathbb{Z}$ is the mode number. For each mode, the scattered field is then given by

$$
u_{m}^{\mathrm{ref}}(r, \theta)=-\frac{J_{m}{ }^{\prime}(\kappa R)}{H_{m}^{(1)^{\prime}}(\kappa R)} H_{m}^{(1)}(\kappa r) e^{\imath m \theta}, \quad r \geq R, \quad m \in \mathbb{Z} .
$$

This solution corresponds to a traveling wave $(m<k)$ or an evanescent wave $(m>k)$. The intermediate region $(m \approx k)$ corresponds to grazing waves. The three kinds of waves are represented in Figure 3 for $R=1$ and $\kappa=25$.

In Figure 4, we represent the relative $L^{2}$-error on the numerical solution and the relative projection $L^{2}$-error as a function of the mode number $m$ for a mesh corresponding to $n_{\lambda}=10$. Let us note that the relative projection error rises with the mode number in the region of the evanescent modes. As the characteristic length of the oscillations along the cylinder decreases, the mesh is too coarse to accurately represents these oscillations. Nevertheless, in the complete plane-wave solution, the coefficients corresponding to the evanescent modes get smaller, and they do not contribute too much to the global error (see e.g. [55])

We observe on Figure 4 that the error corresponding to the evanescent modes does not decrease as much when increasing $N$ for $\phi=0$. It is indeed well-known that the Padé-type HABC is not suited for these modes. Taking $\phi=\pi / 3$ instead of $\phi=0$ decreases the error for these modes, but the error corresponding to the traveling modes rises. 

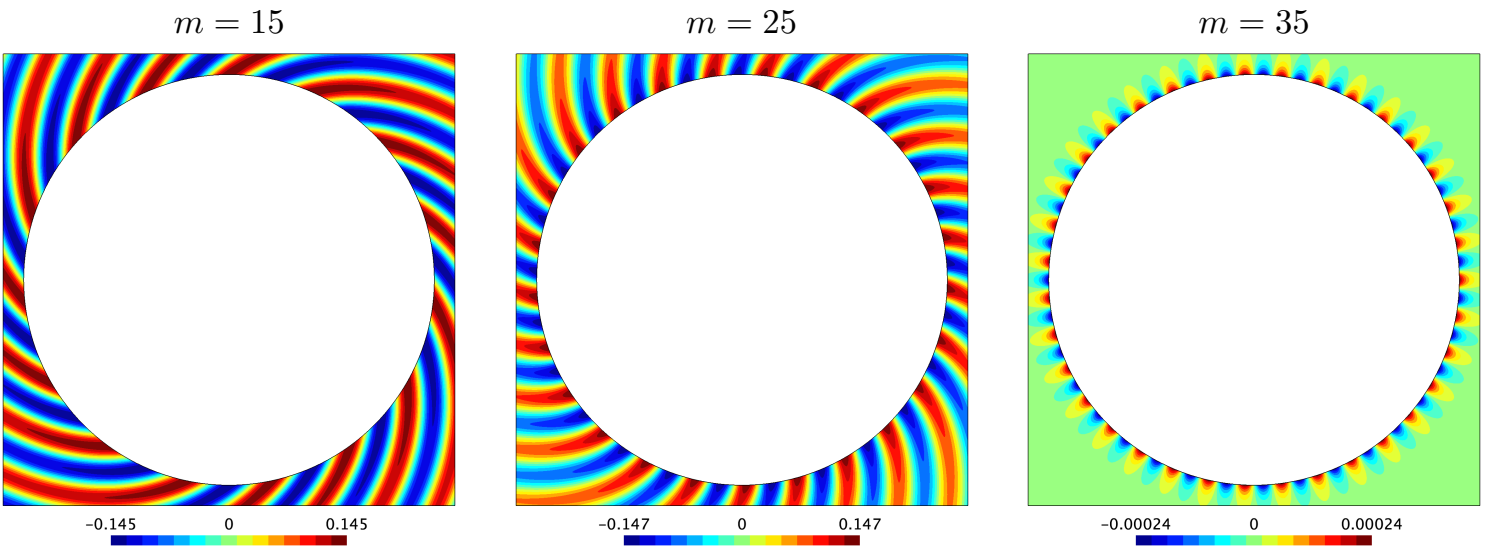

Figure 3: Scattering by a sound-hard circular cylinder: real part of the reference scattered field for a traveling mode $(m=15)$, a grazing mode $(m=25)$ and an evanescent mode $(m=35)$. The radius of the cylinder is $R=1$ and the wavenumber is $\kappa=25$.

(a) $\mathrm{HABC}$ with $\phi=0$

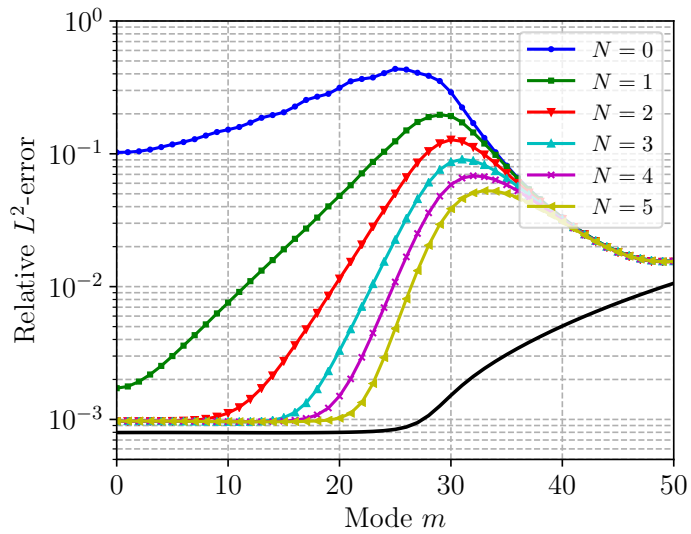

(b) HABC with $\phi=\pi / 3$

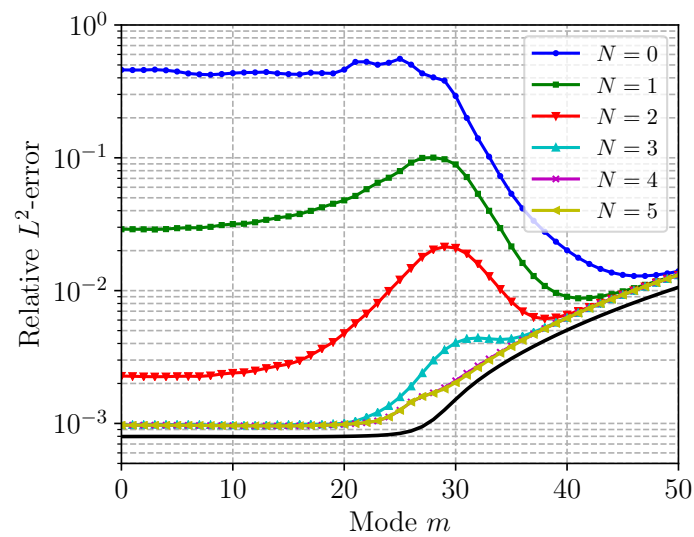

Figure 4: Scattering by a sound-hard circular cylinder: mode-by-mode error for the wave number $\kappa=25$ and the mesh with $n_{\lambda}=10$, which is the third mesh used in the figure 2. In both Figures, the black line corresponds to the relative projection $L^{2}$-error.

\section{Numerical verification in three dimensions}

In order to check our approach in three dimensions, we consider the scattering of a plane wave $u^{\text {inc }}(\vec{x})=$ $e^{\imath \vec{\kappa} \cdot \vec{x}}$ by a sound-hard sphere of radius $R$ centered at the origin. The generated scattered field reads

$$
u^{\mathrm{ref}}(\vec{x})=-\sum_{m=0}^{\infty} i^{m}(2 m+1) \frac{j_{m}{ }^{\prime}(\kappa R)}{{h_{m}{ }^{(1)^{\prime}}(\kappa R)}^{\prime}(1)} h_{m}^{(1)}(\kappa r) P_{m}(\hat{\vec{\kappa}} \cdot \hat{\vec{x}}), \quad r \geq R,
$$

where $\kappa=\|\vec{\kappa}\|, r=\|\vec{x}\|, \hat{\vec{\kappa}}=\vec{\kappa} /\|\vec{\kappa}\|, \hat{\vec{x}}=\vec{x} /\|\vec{x}\|, j_{m}$ is the $m^{\text {th }}$-order spherical Bessel function, $h_{m}^{(1)}$ is the $m^{\text {th }}$-order first-kind spherical Hankel function, and $P_{m}$ is the $m^{\text {th }}$-order Legendre polynomial.

Numerical simulations are performed on the cubic computational domain $\Omega=[-1.41,1.41]^{3}$ with a scattering sphere of radius $R=1$. The HABC is prescribed on all the faces of the cube, and the compatibility relations are used on all the edges and all the corners. The simulation parameters are $\kappa=10$ and $\hat{\vec{\kappa}}=[1 / \sqrt{2}, 1 / \sqrt{2}, 0]$. With this setting, the incident plane wave enters in the computational domain first by hitting an edge of the cube. The mesh is made of second-order curvilinear tetrahedral elements, and second-degree basis functions are used. A snapshot of the reference scattered field is shown on Figure 5a.

The relative $L^{2}$-error between the numerical solution and the reference analytic solution is given for two meshes and different values of the parameters $N$ and $\phi$ in Table 1. For both meshes, increasing the 
(a) Reference solution

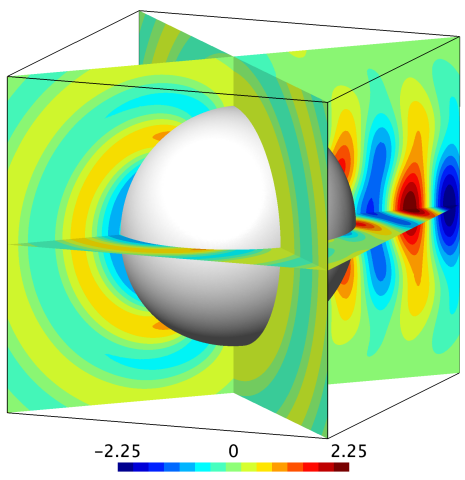

(b) Error with $\phi=0$

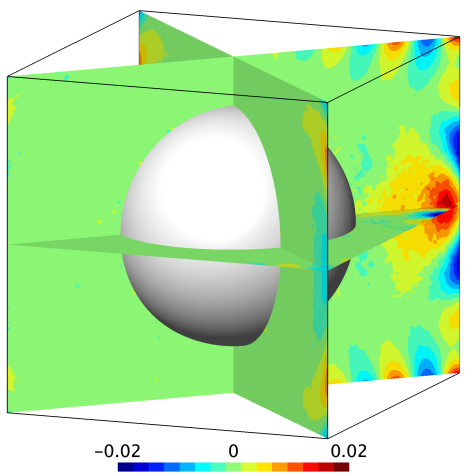

(c) Error with $\phi=\pi / 4$

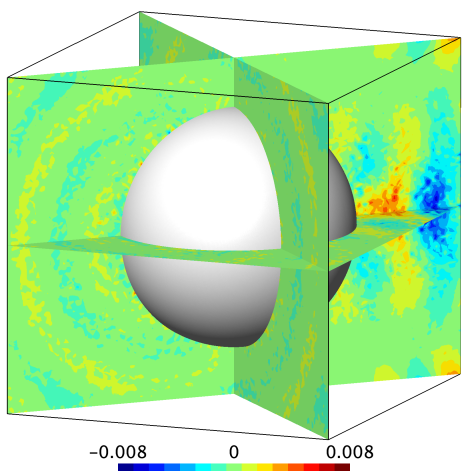

Figure 5: Scattering by a sound-hard sphere: real part of the reference solution $\Re e\left\{u^{\text {ref }}\right\}$ (Figure 5a) and real part of the error $\Re e\left\{u^{\text {num }}-u^{\text {ref }}\right\}$ when using the HABC with the exact corner treatment, $N=3$ and either $\phi=0$ (Figure $5 \mathrm{~b}$ ) or $\phi=\pi / 4$ (figure $5 \mathrm{c}$ ). The mesh is made of 347,592 elements and 501,836 second-order nodes, which corresponds to $n_{\lambda} \approx 10$.

Table 1: Scattering by a sound-hard sphere: relative $L^{2}$-error between the numerical solution and the reference analytic solution (24) for two mesh densities $\left(n_{\lambda}\right)$, different numbers of auxiliary fields $(N)$ and different rotating angles $(\phi)$. The relative projection errors of the exact solution on the meshes are $1.3810^{-3}$ (for $n_{\lambda}=10$ ) and $0.710^{-3}$ (for $n_{\lambda}=12.5$ ). The last column gives the number of degrees of freedom (DoF) for each simulation, and the percentage of \#DoF corresponding to the auxiliary fields.

\begin{tabular}{rl|rrrrr|r} 
& & $\phi=0$ & $\phi=\pi / 8$ & $\phi=\pi / 4$ & $\phi=\pi / 3$ & $\phi=\pi / 2$ & $\#$ DoF (\% in HABC) \\
\hline \multirow{4}{*}{$n_{\lambda}=10$} & $N=1$ & $15.1510^{-3}$ & $8.9110^{-3}$ & $8.4610^{-3}$ & $16.3510^{-3}$ & $60.7510^{-3}$ & $566,118(12 \%)$ \\
& $N=2$ & $3.8110^{-3}$ & $2.3310^{-3}$ & $2.1910^{-3}$ & $2.3710^{-3}$ & $9.2210^{-3}$ & $637,980(22 \%)$ \\
& $N=3$ & $2.5810^{-3}$ & $2.2110^{-3}$ & $2.1810^{-3}$ & $2.1810^{-3}$ & $2.5710^{-3}$ & $712,170(30 \%)$ \\
\hline \multirow{2}{*}{$n_{\lambda}=12.5$} & $N=1$ & $15.0210^{-3}$ & $8.5910^{-3}$ & $8.1610^{-3}$ & $16.2710^{-3}$ & $60.8210^{-3}$ & $1,044,007(10 \%)$ \\
& $N=2$ & $3.5110^{-3}$ & $1.5010^{-3}$ & $1.1210^{-3}$ & $1.4310^{-3}$ & $9.0010^{-3}$ & $1,151,517(18 \%)$ \\
& $N=3$ & $1.6710^{-3}$ & $1.1210^{-3}$ & $1.0810^{-3}$ & $1.0810^{-3}$ & $1.7410^{-3}$ & $1,261,883(26 \%)$ \\
\hline
\end{tabular}

number of auxiliary fields $N$ decreases the relative error until a plateau (at approximatively $2.210^{-3}$ and $1.110^{-3}$, respectively), whatever the value of $\phi$. This plateau corresponds to a numerical error, which is at the same order of magnitude as the relative projection error of the exact solution $\left(1.3810^{-3}\right.$ and $0.710^{-3}$, respectively). In practice, $N$ should be taken as small as possible, since increasing the number of auxiliary fields significantly increases the number of degrees of freedom, as well as the computational cost. For both meshes, the values $N=2$ and $\phi=\pi / 4$ are nearly optimal. With only two auxiliary fields per face, the relative error is close to the one obtained with three auxiliary fields per face. Further refining the mesh leads to a lower error plateau, for which the optimal number of auxiliary fields $N$ will increase.

Snapshots of the error on the numerical solutions obtained with $N=2$ auxiliary fields and the rotating angle $\phi$ equal to 0 and $\pi / 4$ are shown on Figures $5 \mathrm{~b}$ and $5 \mathrm{c}$, respectively. The error is dominated by the dispersion numerical error in the former case, with $\phi=\pi / 4$. Similarly to the two-dimensional case, the error with $\phi=0$ exhibits spurious surface waves propagating along artificial boundaries, in addition to the dispersion numerical error. Note that we observe spurious surface waves also in the $\phi=\pi / 4$ case, close to the corners on the right of figure 5c. Nevertheless, the amplitude of these waves is small in comparison with the dispersion error, and they disappear when increasing the number of auxiliary fields $N$ from 2 to 3 (results not shown here).

These results confirm the effectiveness of both the HABC and the compatibility relations for dealing with the edges and the corners of computational domains with right angles. The strategy with the rotating parameter $\phi$ is very attractive since it improves the quality of the solution at no additional computational cost. 


\section{Approximate treatments for settings with non-right angles}

In order to deal with generally-shaped convex computational domains, corner and edge treatments must be proposed for settings with non-right angles. Unfortunately, the compatibility relations derived in the previous section cannot be straightforwardly extended to more general settings. Indeed, when deriving these relations, we use the fact that the normal derivative for one border is a tangential derivative for the others (see Section 3.1), which does not hold in the general case.

In this section, we propose alternative strategies based on approximate conditions (Section 4.1) and regularization techniques (Section 4.2). The effectiveness and the accuracy of these approaches is analyzed in numerical comparisons (Section 4.3). In contrast with the right-angle compatibility relations of the previous section, which do not involve any additional approximation, the strategies proposed hereafter are approximate treatments, which generate additional modeling error at corners and edges.

\subsection{Approximate conditions at corners and edges}

To describe the corner treatments, we consider the two-dimensional problem defined on the infinite wedge domain $\Omega=\left\{(x, y) \in \mathbb{R}^{2}: x^{a}<0\right.$ and $\left.y^{b}<0\right\}$, where $\left(x^{a}, y^{a}\right)$ and $\left(x^{b}, y^{b}\right)$ are Cartesian coordinate systems associated with the edges $\Gamma^{a}$ and $\Gamma^{b}$, respectively, such as $x^{a}$ and $y^{b}$ are varying along the outward unit normals to the edges, as shown on Figure 6. The angle between both edges is denoted $\alpha$. The HABCs prescribed on both edges can then be written as

$$
\begin{array}{ll}
\left.\partial_{x^{a}} u\right|_{\Gamma^{a}}=\imath \kappa e^{\imath \phi / 2}\left[\left.u\right|_{\Gamma^{a}}+\frac{2}{M} \sum_{i=1}^{N} c_{i}\left(\left.u\right|_{\Gamma^{a}}+\varphi_{i}^{a}\right)\right], & \text { on } \Gamma^{a}, \\
\left.\partial_{y^{b}} u\right|_{\Gamma^{b}}=\imath \kappa e^{\imath \phi / 2}\left[\left.u\right|_{\Gamma^{b}}+\frac{2}{M} \sum_{j=1}^{N} c_{j}\left(\left.u\right|_{\Gamma^{b}}+\varphi_{j}^{b}\right)\right], & \text { on } \Gamma^{b},
\end{array}
$$

where auxiliary fields $\varphi_{i}^{a}$ and $\varphi_{j}^{b}$, defined respectively on $\Gamma^{a}$ and $\Gamma^{b}$, are governed by

$$
\begin{aligned}
& \partial_{y^{a} y^{a}}^{2} \varphi_{i}^{a}+\kappa^{2} e^{\imath \phi}\left(\left(c_{i}+e^{-\imath \phi}\right) \varphi_{i}^{a}+\left.\left(c_{i}+1\right) u\right|_{\Gamma^{a}}\right)=0, \quad \forall i, \quad \text { on } \Gamma^{a}, \\
& \partial_{x^{b} x^{b}}^{2} \varphi_{j}^{b}+\kappa^{2} e^{\imath \phi}\left(\left(c_{j}+e^{-\imath \phi}\right) \varphi_{j}^{b}+\left.\left(c_{j}+1\right) u\right|_{\Gamma^{b}}\right)=0, \quad \forall j, \quad \text { on } \Gamma^{b} .
\end{aligned}
$$

We seek a treatment to prescribe at the corner $P=(0,0)$ for any angle $\alpha \in] 0, \pi]$.

Because of the spatial derivatives in equations (27)-(28), boundary conditions should be prescribed on the auxiliary fields at the corner. Deriving compatibility relations for any angle $\alpha$, as it was done in the previous section for $\alpha=\pi / 2$, would be the ideal solution. However, because we took advantage of the isotropy of the Laplace operator in Cartesian coordinates when deriving the relations for $\alpha=\pi / 2$ (the normal derivative for one border is a tangential derivative for the others), this procedure cannot be straightforwardly applied for non-right corners. To the best of our knowledge, compatibility relations have never been explicitly obtained for $\alpha \neq \pi / 2$. Nevertheless, the relations corresponding to $\alpha=\pi / 2$ can be tested in settings with non-right angles. The solution should be still accurate if the angles are nearly right (i.e. $\alpha \approx \pi / 2$ ).

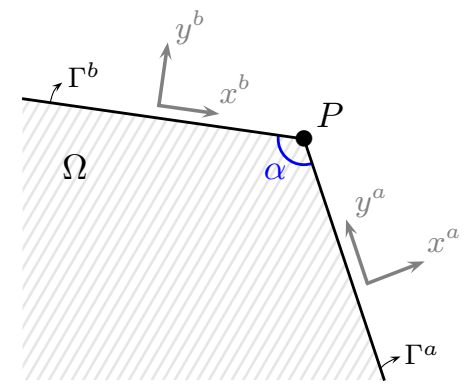

Figure 6: Infinite wedge domain with the coordinate systems. 
As an alternative corner condition, we also propose to prescribe the Sommerfeld boundary condition on the auxiliary fields at the corner,

$$
\begin{aligned}
& \left.\partial_{y^{a}} \varphi_{i}^{a}\right|_{P}=\left.\imath \kappa \varphi_{i}^{a}\right|_{P}, \quad \forall i, \quad \text { at } P, \\
& \left.\partial_{x^{b}} \varphi_{j}^{b}\right|_{P}=\left.\imath \kappa \varphi_{j}^{b}\right|_{P}, \quad \forall j, \quad \text { at } P \text {. }
\end{aligned}
$$

Using such a low-order ABC makes sense since the exact compatibility treatment for $\alpha=\pi / 2$ finally corresponds to using the HABC directly on the auxiliary fields at the corner (see Section 3.1). This treatment is an approximation, even for $\alpha=\pi / 2$, but the computational cost is smaller than with the compatibility relations since the auxiliary fields are not longer coupled.

In three dimensions, when using the $\mathrm{HABC}$ on the faces of polyhedral domains, supplementary boundary conditions must be prescribed at the edges for the auxiliary fields belonging to the faces. Similarly to the two-dimensional case, several approximate conditions are tested for settings with nonright angles. Considering two faces $\Gamma^{a}$ and $\Gamma^{b}$ and the edge $\Upsilon^{a b}=\overline{\Gamma^{a}} \cap \overline{\Gamma^{b}}$, we propose:

1. using the Sommerfeld boundary condition on the auxiliary fields at the edge,

$$
\begin{aligned}
& \left.\partial_{y^{a}} \varphi_{i}^{a}\right|_{\Upsilon^{a b}}=\left.\imath \kappa \varphi_{i}^{a}\right|_{\Upsilon^{a b}}, \quad \forall i, \quad \text { at } \Upsilon^{a b}, \\
& \left.\partial_{x^{b}} \varphi_{j}^{b}\right|_{\Upsilon^{a b}}=\left.\imath \kappa \varphi_{j}^{b}\right|_{\Upsilon^{a b}}, \quad \forall j,
\end{aligned}
$$

where the auxiliary fields $\varphi_{i}^{a}$ and $\varphi_{j}^{b}$ are defined on $\Gamma^{a}$ and $\Gamma^{b}$, respectively, and the coordinates $y^{a}$ and $x^{b}$ are varying along the outward unit normal to the boundary of these faces, on the edge $\Upsilon^{a b}$;

2. using the two-dimensional right-angle compatibility relation,

$$
\begin{aligned}
& \left.\partial_{y^{a}} \varphi_{i}^{a}\right|_{\Upsilon^{a b}}=\mathcal{L}\left(\left.\varphi_{i}^{a}\right|_{\Upsilon^{a b}}, \varphi_{i 1}^{a b}, \ldots, \varphi_{i N}^{a b}\right), \quad \forall i, \quad \text { at } \Upsilon^{a b}, \\
& \left.\partial_{x^{b}} \varphi_{j}^{b}\right|_{\Upsilon^{a b}}=\mathcal{L}\left(\left.\varphi_{j}^{b}\right|_{\Upsilon^{a b}}, \varphi_{1 j}^{a b}, \ldots, \varphi_{N j}^{a b}\right), \quad \forall j, \quad \text { at } \Upsilon^{a b},
\end{aligned}
$$

with auxiliary fields defined on $\Upsilon^{a b}$ and given by

$$
\varphi_{i j}^{a b}=-\frac{\left.\left(c_{j}+1\right) \varphi_{i}^{a}\right|_{\Upsilon^{a b}}+\left.\left(c_{i}+1\right) \varphi_{j}^{b}\right|_{\Upsilon^{a b}}}{c_{i}+c_{j}+e^{-\imath \phi}}, \quad \forall i, j, \quad \text { at } \Upsilon^{a b}
$$

3. using the three-dimensional right-angle compatibility relation, which leads to equations (29)-(30), with auxiliary fields on $\Upsilon^{a b}$ governed by

$$
\partial_{z z} \varphi_{i j}^{a b}+\kappa^{2} e^{\imath \phi}\left(\left(c_{i}+c_{j}+e^{-\imath \phi}\right) \varphi_{i j}^{a b}+\left.\left(c_{j}+1\right) \varphi_{i}^{a}\right|_{\Upsilon^{a b}}+\left.\left(c_{i}+1\right) \varphi_{j}^{b}\right|_{\Upsilon^{a b}}\right)=0, \quad \forall i, j, \quad \text { on } \Upsilon^{a b},
$$

where the coordinate $z$ is varying along $\Upsilon^{a b}$.

In the latter case, boundary conditions are required at the corners for the auxiliary fields living on the edges and governed by equation (31). We propose using the Sommerfeld condition or, if only three edges meet at the corner, using the three-dimensional right-angle compatibility relation. Therefore, we finally have four strategies with different computational costs. The first strategy is the cheapest one, while using compatibility relations on both edges and corners is the most expensive one.

\subsection{Treatment by regularization of boundary}

Another approach for dealing with domains having corners and edges consists in defining the HABC on a regularized boundary: the sharp corners are replaced with rounded corners (see illustration on Figure 7), avoiding the need for auxiliary conditions. Then, a HABC for curved boundaries is used as is for computing the numerical solution on the original mesh with the sharp corners, which obviously constitutes an approximation. The numerical curvature effect should reproduce in a heuristic way the wave propagation at the corner. 


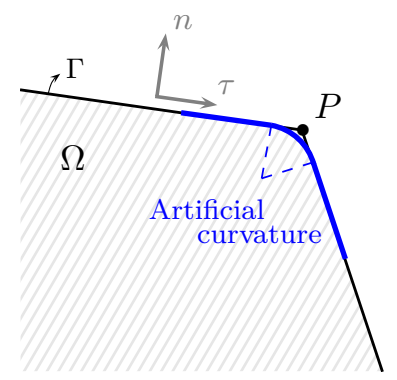

Figure 7: Infinite wedge domain and illustration of the regularization approach.

\section{HABC for regular curved boundaries}

The Padé-type HABC derived in Section 2 is suited only for planar boundaries, since it approximates the non-local boundary condition with the DtN operator (1) which is exact only for planar boundaries. Deriving such a DtN operator for curved boundaries is challenging. In [5], Antoine, Barucq and Bendali have derived the first terms of an expansion of the symbol of the DtN operator for regular curved surfaces. The first term of the expansion corresponds to the total symbol in the planar case (with the square root), while the curvature appears in the other terms. This expansion has been used to propose families of local ABCs for curved surfaces [3, 5], which have been applied to finite element simulations [72]. Unfortunately, these ABCs cannot model evanescent waves, because the square root in the first term has been approximated using a low-order Taylor expansion [8]. To improve the accuracy of the solution, Kechroud, Antoine and Soulaimani [53] have combined the Padé-type approximation for the square root (equation (3)) and using few additional terms of the expansion to take curvature effects into account.

In two dimensions, the modified $\mathrm{HABC}$ for a regular planar curve $\Gamma \subset \mathbb{R}^{2}$ obtained in [53] reads

$$
\left.\partial_{n} u\right|_{\Gamma}=\imath \kappa e^{\imath \phi / 2}\left[\left.u\right|_{\Gamma}+\frac{2}{M} \sum_{i=1}^{N} c_{i}\left(\left.u\right|_{\Gamma}+\varphi_{i}\right)\right]-\left.\frac{\gamma}{2} u\right|_{\Gamma}+\left.\frac{\gamma^{2}}{8(\gamma-\imath \kappa)} u\right|_{\Gamma}-\partial_{\tau}\left(\left.\frac{\gamma}{2 k^{2}} \partial_{\tau} u\right|_{\Gamma}\right), \quad \text { on } \Gamma,
$$

with the auxiliary fields $\varphi_{i}$ defined on $\Gamma$ and governed by

$$
\partial_{\tau \tau}^{2} \varphi_{i}+\kappa_{\varepsilon}^{2} e^{\imath \phi}\left(\left(c_{i}+e^{-\imath \phi}\right) \varphi_{i}+\left.\left(c_{i}+1\right) u\right|_{\Gamma}\right)=0, \quad \forall i, \quad \text { on } \Gamma,
$$

where $n$ and $\tau$ are the coordinates in the normal and tangent directions on $\Gamma$ (see Figure 7 ), $\partial_{n}$ and $\partial_{\tau}$ are the normal and tangential derivatives, $\gamma(\tau)$ is the curvature, and $\kappa_{\varepsilon}=\kappa+\imath \varepsilon$ is a modified wavenumber. Using the same strategy for a regular surface $\Gamma \subset \mathbb{R}^{3}$ in three dimensions, we have the HABC

$$
\left.\partial_{n} u\right|_{\Gamma}=\imath \kappa e^{\imath \phi / 2}\left[\left.u\right|_{\Gamma}+\frac{2}{M} \sum_{i=1}^{N} c_{i}\left(\left.u\right|_{\Gamma}+\varphi_{i}\right)\right]-\left.\mathcal{H} u\right|_{\Gamma}-\operatorname{div}_{\Gamma}\left(\frac{\mathcal{H}}{2 k^{2}} \nabla_{\Gamma} u\right), \quad \text { on } \Gamma,
$$

with auxiliary fields $\varphi_{i}$ governed by

$$
\Delta_{\Gamma} \varphi_{i}+\kappa_{\varepsilon}^{2} e^{\imath \phi}\left(\left(c_{i}+e^{-\imath \phi}\right) \varphi_{i}+\left.\left(c_{i}+1\right) u\right|_{\Gamma}\right)=0, \quad \forall i, \quad \text { on } \Gamma,
$$

where $\mathcal{H}$ is the mean curvature of $\Gamma$. These boundary conditions are easily incorporated in the finite element scheme using the variational formulation described in [53] for the two-dimensional case.

In comparison with the planar HABC, additional terms have been added in equations (32) and (34), and an imaginary part has been added to the wavenumber in the auxiliary equations (33) and (35). Using a complex wavenumber significantly improves the accuracy of the solution [8], which is a very attractive strategy since there is nearly no additional cost. Optimized choices of the imaginary damping part $\varepsilon$ stated in $[8,30]$ are $\varepsilon \approx 0.4 \kappa^{1 / 3} \gamma^{2 / 3}$ (in two dimensions) and $\varepsilon \approx 0.4 \kappa^{1 / 3} \mathcal{H}^{2 / 3}$ (in three dimensions).

In particular cases where the boundary is straight, the curvature (or the main curvature in three dimensions) is zero, and the HABC for straight boundaries is recovered. Let us emphasize that, in equation (32), there is only one set of auxiliary fields, which are continuous at the corner. By contrast, 
in equations (25) and (26), the auxiliary fields constitute two sets of fields, each of them corresponding to one edge.

In the remainder, using the $\mathrm{HABC}$ for straight boundaries with continuous auxiliary fields at a corner is called hard regularization of the boundary, while using the $\mathrm{HABC}$ with a numerical curvature is called soft regularization of the boundary.

\section{Numerical curvature and numerical mean curvature}

Selecting a numerical curvature $\gamma_{\text {num }}$ or a numerical mean curvature $\mathcal{H}_{\text {num }}$ is a tricky step, which directly impacts the accuracy of the solution. In this work, they are obtained by solving auxiliary problems on the boundary with a finite element method, as a pre-processing operation.

In two dimensions, the curvature verifies the Frenet formula $\partial_{\tau} \vec{n}=\gamma \vec{t}$, where $\vec{n}(\tau)$ and $\vec{t}(\tau)$ are the outward unit normal and the unit tangent associated with $\tau$. First, we compute the $L^{2}$-projection of $\vec{n}$ onto $\left(X_{N}\right)^{2}$, where $X_{N}$ is a scalar finite element space defined on the border of the mesh. While $\vec{n}$ is discontinuous at the corner, its $L^{2}$-projection $\vec{n}_{\text {proj }}$ is continuous. Then, the numerical curvature is computed by solving the following variational problem: Find $\gamma_{\text {num }} \in X_{N}$ such that

$$
\int_{\Gamma}\left(\gamma_{\text {num }}-\vec{t} \cdot \partial_{\tau} \vec{n}_{\text {proj }}\right) \psi=0, \quad \forall \psi \in X_{N}
$$

In three dimensions, the main curvature is computed by solving weakly the formula $2 \mathcal{H}=-\nabla \cdot \tilde{\vec{n}}$, where $\tilde{\vec{n}}$ is an extension of the normal in a neighborhood of the surface [33]. Similarly to the two-dimensional case, we use the following variational problem: Find $\mathcal{H}_{\text {num }} \in X_{N}$ such that

$$
\int_{\Gamma}\left(2 \mathcal{H}_{\text {num }}+\vec{t}_{1} \cdot \partial_{\tau_{1}} \vec{n}_{\text {proj }}+\vec{t}_{2} \cdot \partial_{\tau_{2}} \vec{n}_{\text {proj }}\right) \psi=0, \quad \forall \psi \in X_{N},
$$

where $\vec{t}_{1}$ and $\vec{t}_{2}$ are two perpendicular unit tangents, and $\tau_{1}$ and $\tau_{2}$ are the associated coordinates. In practice, $\vec{n}_{\text {proj }}, \gamma_{\text {num }}$ and $\mathcal{H}_{\text {num }}$ are computed by using linear basis functions on the boundary of the mesh generated for solving the Helmholtz problem.

The numerical evaluation of curvature and mean curvature is already used for simulations with domains having regular borders (see e.g. $[1,20,30]$ ). Using this approach for domains having corners was suggested in $[2,3]$. However, to the best of our knowledge, the accuracy of the resulting schemes has never been studied. Let us mention that the curvature can also be defined with heuristic formula (see e.g. $[3,19,60]$ ). In preliminary simulations, the formula we have tested gave, at the best, the same level of accuracy as with the approach described above. The careful comparison of these formulas is let for future works.

\subsection{Numerical comparison of the corner treatments}

To study and to compare the accuracy of the corner treatments for different angles, we again use the scattering problems described in Section 3.3: the scattering of a plane wave by a disk or a sphere. Here, we consider different computational domains having non-right corners: regular polygons, slices of a disk, and regular polyhedra. The two-dimensional settings are shown on Figure 8.

\section{Polygonal truncated domains}

In the first setting, a scattering disk of radius $R=1$ is placed in the center of a regular polygon, which the midradius is 1.65, as shown on Figure 8a for the hexagon case. The HABC is prescribed on all the sides of the polygon, and the same corner treatment is used for all the corners. The simulation parameters are the same as in Section 3.3, with the wavenumber $\kappa=25$, the number of vertices per wavelength $n_{\lambda}=10$ and second-order nodal finite elements. We use $N=4$ auxiliary fields per edge and the rotating angle $\phi=\pi / 3$. Note that increasing $N$ does not significantly change the results.

Snapshots of the error in the triangular case are shown on Figure 9 for the different corner treatments: using the $\mathrm{HABC}$ with continuous auxiliary fields at the corners (hard regularization), using the HABC for curved boundaries without and with the three last terms in equation (32) (soft regularization), using the Sommerfeld condition on the auxiliary fields at the corner, and using the right-angle compatibility relations as an approximate treatment. 
(a) Regular polygon

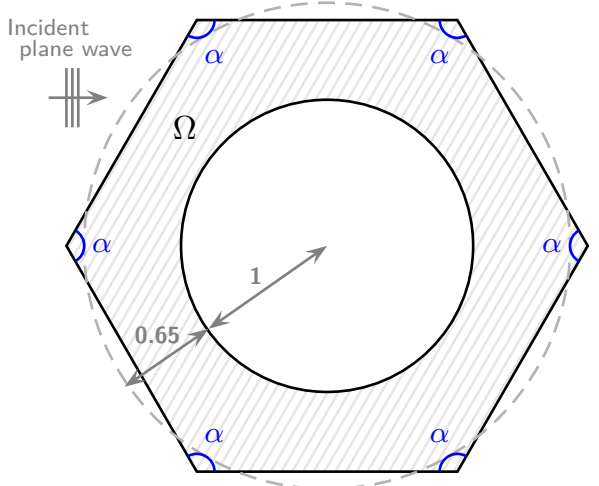

(b) Slice of a disk

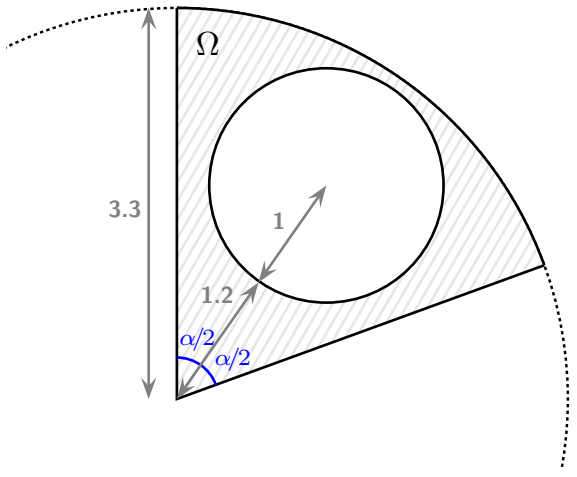

Figure 8: Two-dimensional settings for the comparison of the corner treatments with a varying angle $\alpha$. In Figure $8 \mathrm{a}$, the radius of the the dashed circle is midradius of the polygon.

(a) Reference solution

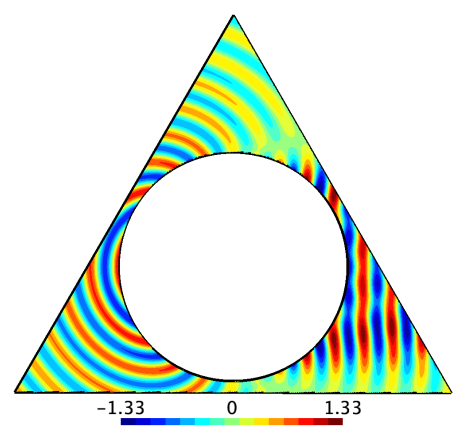

(d) Hard regularization

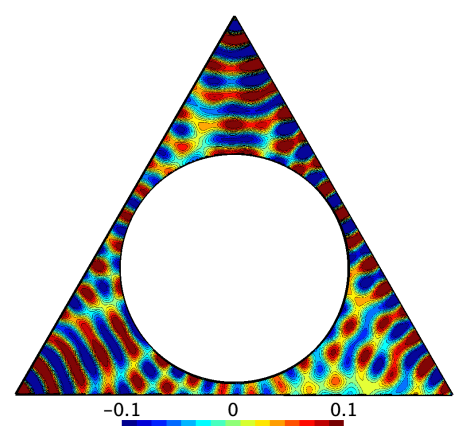

(b) Sommerfeld BC at corners

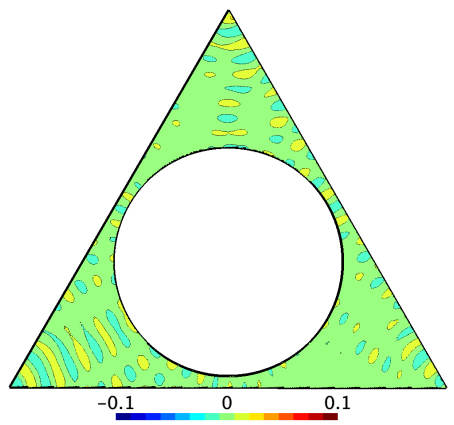

(e) Soft regularization 1

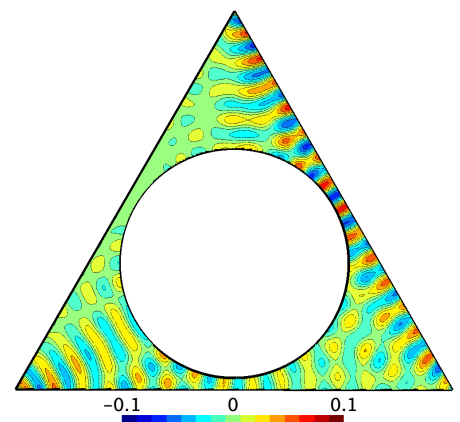

(c) Right-angle compatibility

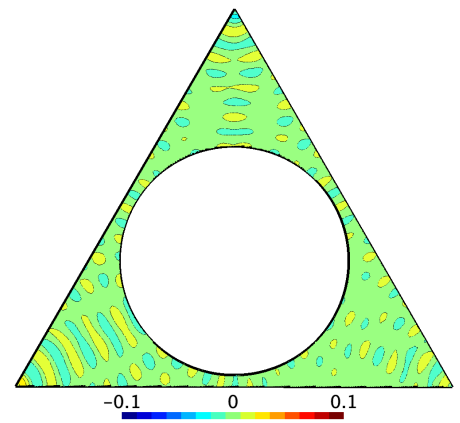

(f) Soft regularization 2

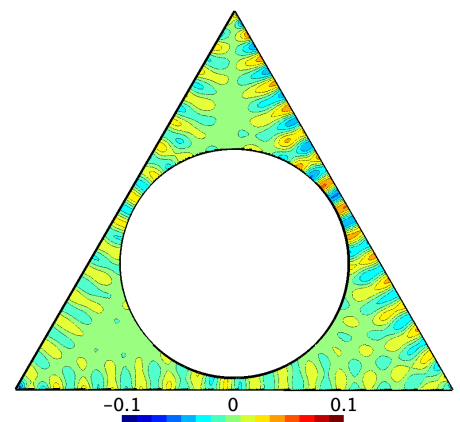

Figure 9: Error analysis for the triangular domain: analytic reference solution $u^{\text {ref }}$ (a) and error on the numerical solutions obtained with the approximate corner conditions (b)-(c) and the regularization strategies (d)-(f). The soft regularization is tested without (e) and with (f) the three last terms in equation (32).

Using the right-angle compatibility relations in this setting with a non-right angle $(\alpha=\pi / 3$ here $)$ introduces an additional error, which is visible on Figure 9c. This error is likely due to the corner treatment, since the error related to the HABC was negligible for a square domain with the same simulation parameters and the same minimum distance between the scattering object and the exterior boundary (see Section 3.3). In addition, the profile of error looks like spurious waves generated at the corners. The error is rather similar when the Sommerfeld condition is used at the corners. It is slightly 
(a) $n_{\lambda}=10$

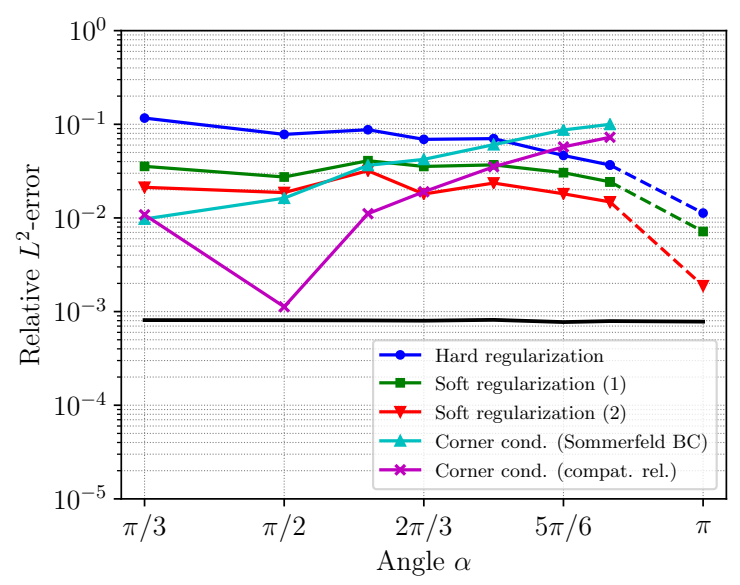

(b) $n_{\lambda}=20$

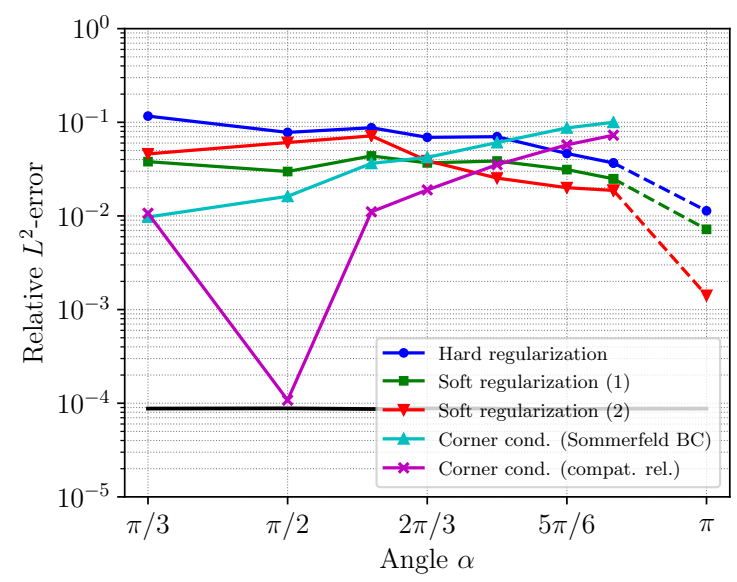

Figure 10: Polygonal truncated domains: Relative $L^{2}$-error of the numerical solution $u$ compared to the reference solution $u^{\text {ref }}$ as a function of the angle $\alpha=\pi-2 \pi / N_{\text {sides }}$ for different corner treatments and two meshes. The numbers of vertices per wavelength $n_{\lambda}$ are 10 (a) and 20 (b). The values for $\alpha=\pi$ have been obtained with a circular domain and the exact curvature. The black line corresponds to the relative $L^{2}$-projection error.

larger with both soft regularization strategies, and it dramatically rises with the hard regularization.

For a quantitative comparison of the corner treatments with a varying angle, the relative $L^{2}$-error of the numerical solution compared to the reference solution (23) has been computed for polygonal domains with $3,4,5,6,8,12$ and 18 sides, and for the circular domain of radius 1.65 . The relative error is plotted as a function of $\alpha$ for the different corner treatments and two different meshes on Figure 10.

For $\alpha=\pi / 3$, both corner conditions provide the lowest relative error. For the right angle, the error obtained with the compatibility relations is very close to the projection error of the exact solution, while the errors obtained with the other strategies is larger by at least one order (for $n_{\lambda}=10$ ) and two orders (for $n_{\lambda}=20$ ). When increasing $\alpha$, the errors for both corner conditions rise, while those for the regularization strategies slowly decrease. For $\alpha>2 \pi / 3$, the soft regularization with the additional terms gives the best results. That technique always performs better than the other regularizations, whatever the angle. The relative errors do not vary much when refining the mesh, except for the compatibility relation in the right-angle case, which confirms that the error is mostly dominated by a modeling error.

\section{Sliced truncated domains}

In order to refine the analysis, we consider the second setting, where the scattering disk is placed inside a slice of a larger disk, as sketched on Figure 8b. The HABC is prescribed on both straight sides of the slice, and the HABC for curved boundaries (32) is used on the exterior circular border with the exact curvature. The compatibility relations are used at both exterior corners, which are right, and the different corner strategies are tested on the interior corner, with a varying angle $\alpha \in[\pi / 3, \pi]$.

While the first setting allows only a discrete set of values for $\alpha$, corresponding to the regular polygons, a continued range of angles can be studied with this setting. However, the HABC prescribed on the curved boundary generates a spurious error, caused by curvature effects, that cannot be reduced simply by increasing the number of auxiliary fields. We observe this error by comparing the snapshots of error for the numerical solutions computed on a slice (Figure 11b) and on the whole disk (Figure 11c, only the part on the slice is shown). For the latter case, the error that is visible is due to the treatment at the curved boundary, since there is no corner in this simulation. In order to isolate the error generated by the corner treatment, we consider the difference between the numerical solution computed on a slice, and the reference numerical solution computed on the whole domain. The difference shown on Figure $11 \mathrm{~d}$ then exhibits only a wave generated at the corner, which is similar to the one observed at the corners of the triangular domain on Figure 9c.

On Figure 12, we show the relative $L^{2}$-errors on the numerical solution as a function the angle $\alpha$ for the different corner strategies. The errors are computed by comparing the numerical solution to 
(a) $\mathcal{R} e\left(u^{\mathrm{ref}}\right)$

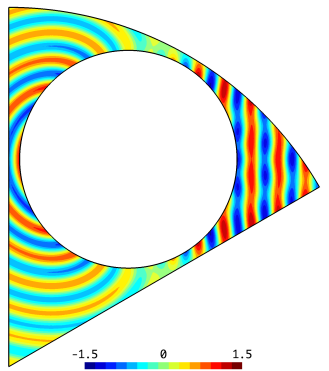

(b) $\mathcal{R} e\left(u-u^{\text {ref }}\right)$

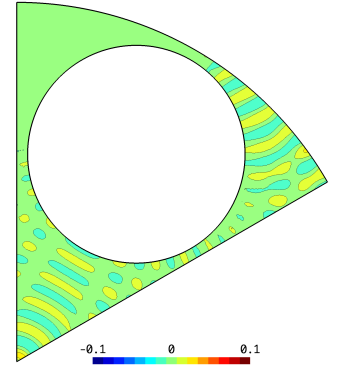

(c) $\mathcal{R} e\left(u^{\text {ref-num }}-u^{\text {ref }}\right)$

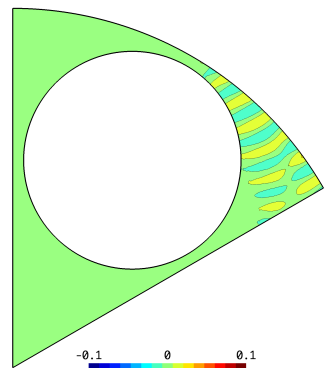

(d) $\mathcal{R} e\left(u-u^{\text {ref.num }}\right)$

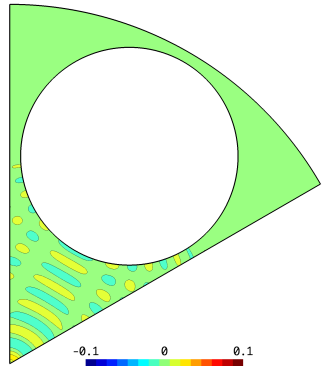

Figure 11: Error analysis for the sliced domain with angle $\alpha=\pi / 3$ : analytic reference solution $u^{\text {ref }}$ (a), error on the numerical solution $u$ when using the approximate compatibility relations at the interior corner (b) and error on the numerical solution $u^{\text {ref-num }}$ computed on the whole disk (c). The difference between both numerical solutions is shown on the last figure (d).

(a) Error versus analytic reference

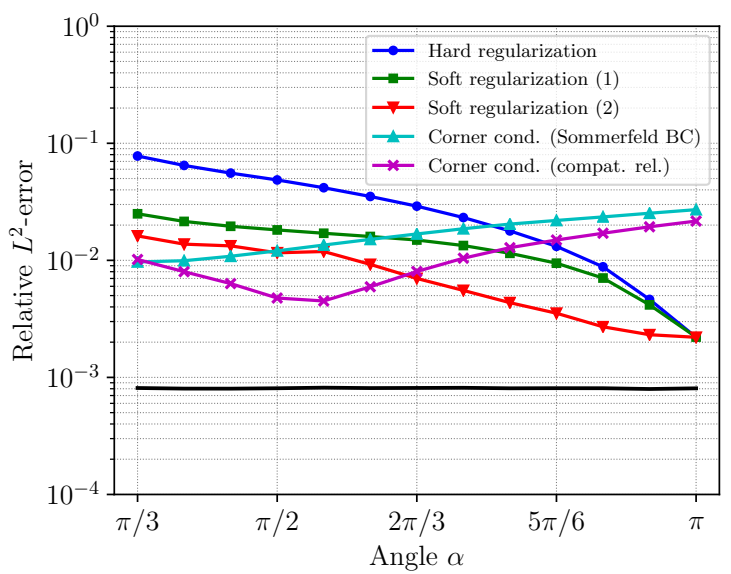

(b) Error versus numerical reference

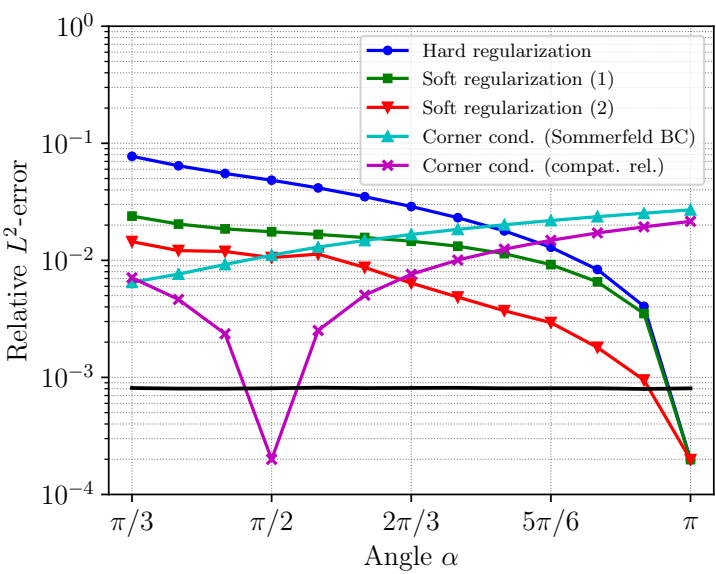

Figure 12: Slided truncated domains: relative $L^{2}$-error on the numerical solution compared to the analytic reference solution $u^{\text {ref }}$ (a) and compared to the numerical reference solution $u^{\text {ref.num }}$ (b) as a function of the angle $\alpha$ with the different corner treatments. The black line corresponds to the relative $L^{2}$-projection error.

the analytic solution (figure 12a) or to the reference numerical solution (figure 12b). For the acutest angle $(\alpha=\pi / 3)$, the corner conditions give the lowest error. It is one order smaller than with the hard regularization. For $\alpha \in[\pi / 3,2 \pi / 3]$, the compatibility relation gives the smallest error. This is the best strategy. For $\alpha>2 \pi / 3$, the best strategy is the second soft regularisation. It provides a relative error smaller than $10^{-2}$, which decreases for larger and larger angles.

The most difficult angles to deal with are $\pi / 3$ and $2 \pi / 3$. For each of them, the best strategy gives a relative error close to $10^{-2}$, which is approximately one order of magnitude larger than the projection error. By contrast, for $\pi / 2$ and $\pi$, the best errors are smaller than the projection error (Figure 12b). In those cases, the best strategies actually are exact corner treatments. Nevertheless, the error grows rapidly when moving away from these very specific angles.

\section{Polyhedral truncated domains}

For the last benchmark, we consider the scattering of a plane wave by a unit sphere placed in the center of a regular polyhedral computational domain. The simulation has been performed for the six Platon solids with the HABC prescribed on each face, and for a spherical domain as a limit case. The midradius of the polyhedra and the radius of the spherical domain are equal to 2 . The numerical setting is the same as in Section 3.3, with $N=2$ auxiliary fields per face and the rotating angle $\phi=\pi / 4$. In all the polyhedral cases, the incident plane wave enters in the truncated domain first by hitting an edge. The 


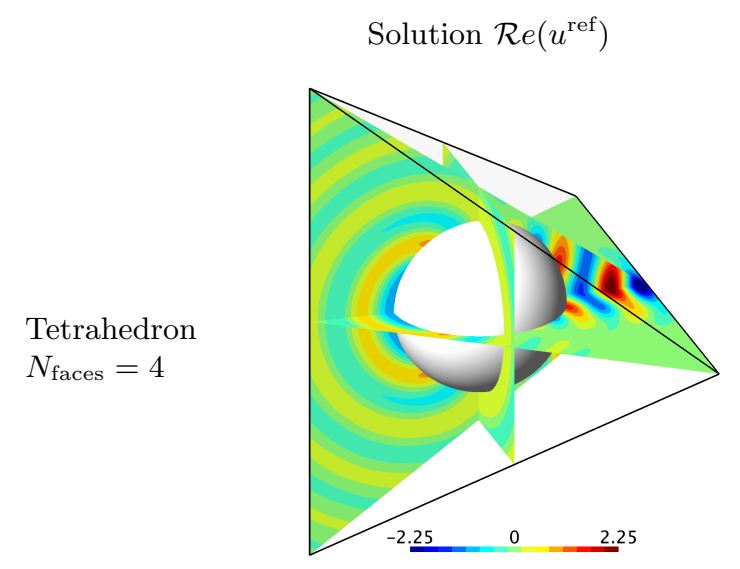

Error $\operatorname{Re}\left(u-u^{\mathrm{ref}}\right)$
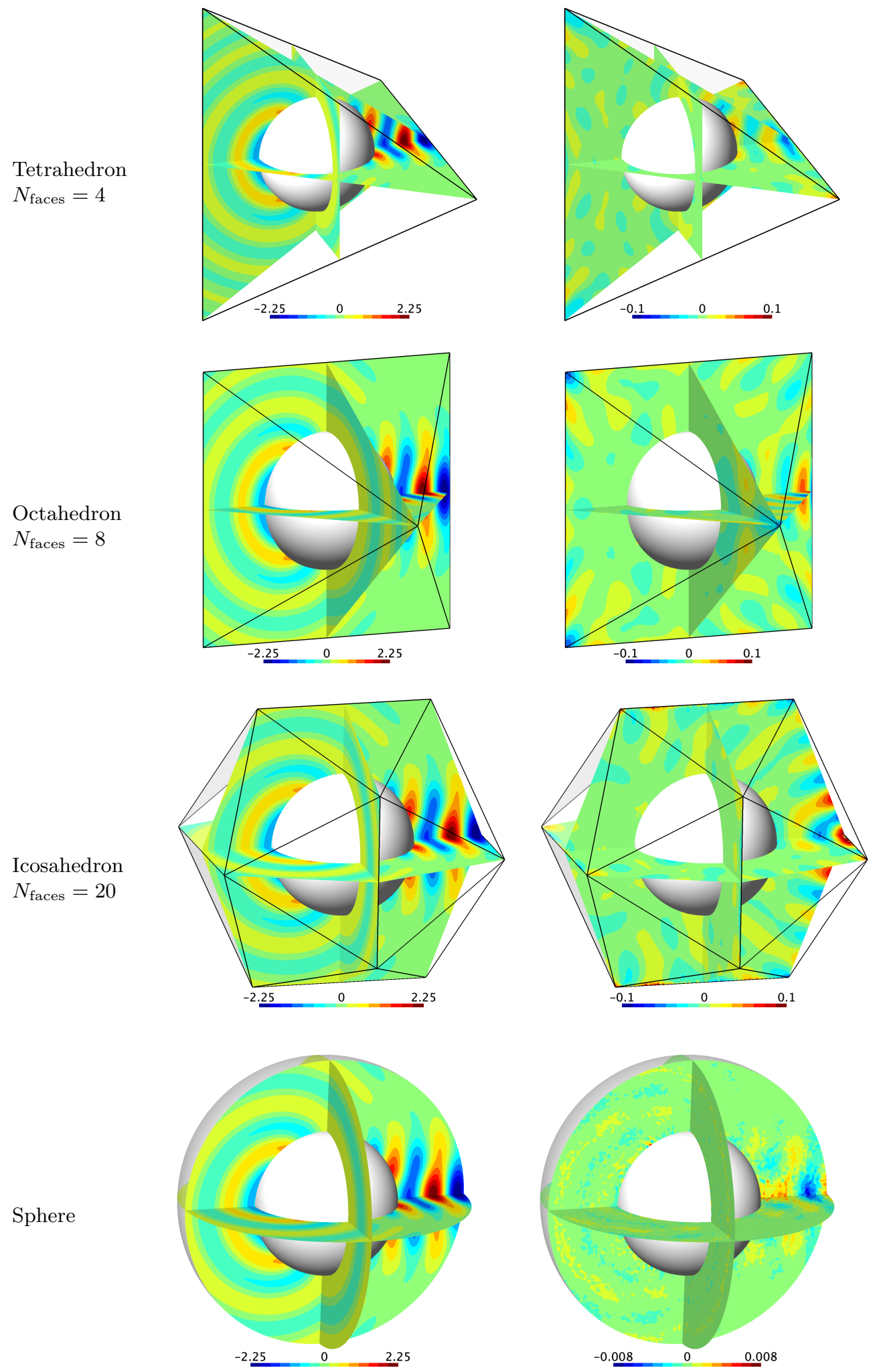

Figure 13: Three-dimensional truncated domains: snapshots of the reference solution and the simulation error for different domains when using the two-dimensional compatibility relations (tetrahedron and octahedron) or the second soft regularization (icosahedron and sphere). The range of the colorbar in the snapshots of error is set to $[-0.1,0.1]$ for the three polyhedra, and the one used in Figure 5 is reused for the sphere. 


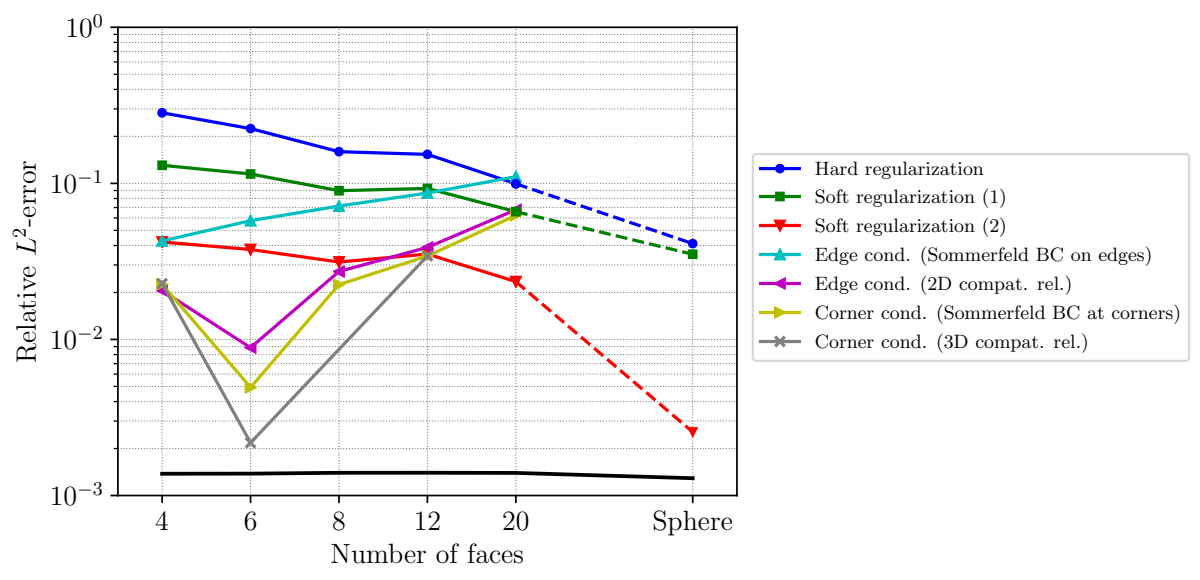

Figure 14: Three-dimensional truncated domains: relative $L^{2}$-error of the numerical solution $u$ compared to the reference solution $u^{\text {ref }}$ as a function of the number of faces for the different corner treatments. The black line corresponds to the relative $L^{2}$-projection error.

real part of the the reference scattered field and the error are shown on Figure 13 for several cases. The setting for the cube exactly corresponds to the one in Section 3.3 .

The relative $L^{2}$-error on the solution is plotted as a function of the number of faces on Figure 14 for the different treatments: the four approximate conditions described in Section 4.1 and the regularization techniques. For the cube and the sphere, the error is very close to the projection error when using the right-angle 3D compatibility relations and the modified $\mathrm{HABC}$ with the curvature, respectively. These techniques are obviously well suited for these limit cases. In the other cases, the approximate conditions perform better for angles closes to $\pi / 2$ (i.e. with a small number of faces), while the regularization approaches give better results for obtuse angles (i.e. with a large number of faces).

Among the approximate conditions, the two-dimensional right-angle compatibility relations at the edges (magenta curve on Figure 14) is the rather attractive. It gives errors similar to those with the three-dimensional compatibility relations prescribed on the edges, whatever the treatment at the corner (yellow curve and black crosses), for $=4,8$ and 12 faces, while it requires less memory storage. The second soft regularization is always the best regularization technique. This validates our approach with the modified HABC having additional terms and a numerical computation of the curvature.

\section{Conclusion}

We have addressed the use of the Padé-type HABC for the finite-element solution of high-frequency scattering problems with polygonal and polyhedral computational domains, offering geometric flexibility when choosing the shape of the domain in application contexts. While this HABC provides a very effective nonreflective treatment for domains with regular boundary, domains having corners and edges require specific care to preserve accuracy.

In this article, two approaches for dealing with corners and edges are proposed and analyzed with numerical benchmarks in two and three dimensions:

- The first approach is based on compatibility relations involving auxiliary fields. Being derived for corners and edges with right angles, they provide a perfect treatment for rectangular and cuboid computational domains. Such relations are not available for settings with non-right angles, but using the right-angle compatibility relations as an approximate treatment for angles close to $\pi / 2$ already provides a good accuracy.

- The second approach consists in regularizing the boundary at corners and edges, and using a HABC for curved boundary with a numerical curvature. This artificial curvature reproduces in a heuristic way the wave propagation at corners and edges. This approach is very effective and overcomes the other approaches for settings with very obtuse angles (with angles close to $\pi$ ). 
In two dimensions, we have observed that using right-angle compatibility relations is the best approach for angles in the range $[\pi / 3,2 \pi / 3]$, and using a soft regularization with a numerical curvature is better in the range $[2 \pi / 3, \pi]$.

These approaches can be applied to deal with other physical waves, such as electromagnetic and elastic waves for which similar Padé-type HABCs have been proposed [20, 30, 61]. They could also be applied in the time domain: the right-angle compatibility relations have already been tested for transient acoustics with cuboidal domains [65].

Our main perspective for this work actually concerns other kind of methods. The Padé-type HABCs are used in acceleration techniques for solving boundary integral solvers [6, 21, 26] and in domain de decomposition methods for parallel finite element solvers [18, 64]. The improvement of these methods thanks to the strategies proposed here is currently investigated.

\section{A Links between different families of high-order absorbing bound- ary conditions}

The Padé-type HABC is related to different HABCs proposed in the literature for planar boundaries. Although the construction processes and the final formulations can be very different, these HABCs are connected to the same general boundary condition. In this appendix, we discuss and establish links between some well-known HABCs.

\section{Different formulations for the HABCs}

Early HABCs have been written with high-order partial derivatives [31, 49], where the highest order of derivative is related to the order of approximation. However, their applicability is limited to the lowest orders because of the high-order derivatives that must be discretized.

In the approach that we have followed, auxiliary fields and auxiliary equations are defined on the boundary to reduce the order of the partial derivatives. This approach has been first used by Collino [23] for a Padé-type HABC based the sum of prime fractions (2). The formulations proposed afterwards are related to approximations of the square root written as sums of prime fractions (e.g. [53, 65]) or as continued fractions (e.g. [36, 37, 40, 42-45, 69, 76]).

With both kinds of representations, auxiliary fields are governed by wave-like equations defined on the boundary (equation (7) in our case). For the representations written with continued fractions, the auxiliary equations are coupled in a recursive way. In specific cases, these conditions can be interpreted as discrete PMLs with discretizations based on finite differences [11, 28] or finite elements [39, 69]. Each auxiliary field then corresponds to an additional layer of the discrete PML. By contrast, for representations written with sums of prime fractions, the auxiliary equations are decoupled. Different representations corresponding to the same approximation of the square root should lead to the same accuracy. The main differences are related to the practical implementation of the final HABCs. HABCs interpreted as discrete PMLs are attractive since they inherit the easiness of implementation from PMLs, with facilities to deal with corners thanks to tensor-product operations at the discrete level. Nevertheless, to the best of our knowledge, this approach has been applied only to low-order discretisations.

\section{Reformulations as continued-fraction HABCs}

In the time-harmonic context, most of these HABCs can be considered as approximate versions of the exact nonreflective boundary condition

$$
\partial_{x} v=\imath \kappa \sqrt{1+\Delta_{\Gamma} / \kappa^{2}} v
$$

with a rational approximation of the square root $f(X)=\sqrt{1+X}$. In order to highlight equivalences between some well-known HABCs, we show that these conditions can be rewritten as

$$
\partial_{x} u=\imath \kappa f_{M}\left(\Delta_{\Gamma} / \kappa^{2}\right) u,
$$


where $f_{M}(X)$ is a continued fraction provided by the general sequence

$$
\begin{aligned}
f_{m}(X) & =\alpha_{m}+\frac{X+1-\alpha_{m}^{2}}{\alpha_{m}+f_{m-1}(X)}, \quad \text { for } m=2 \ldots M, \\
f_{1}(X) & =\alpha_{1},
\end{aligned}
$$

where $\left\{\alpha_{m}\right\}_{m=1 \ldots M}$ are complex parameters. These parameters depend on the kind of HABC.

In section 2.2, the Padé approximation of the square root is written as the sum of prime fractions (2). The $M^{\text {th }}$-order Padé approximation $f_{M}^{\text {Padé }}(X)$ can also be represented as the continued fraction provided by the sequence [32]

$$
\begin{aligned}
& f_{m}^{\text {Padé }}(X)=1+\frac{X}{1+f_{m-1}^{\text {Padé }}(X)}, \quad \text { for } m=2 \ldots M, \\
& f_{1}^{\text {Padé }}(X)=1 .
\end{aligned}
$$

If $M$ is odd and $N=(M-1) / 2$, the continued fraction is rigorously equivalent to the sum of prime fractions (2) (see e.g. $[12,38]$ ). Using this result, the $\phi$-rotated Padé approximation of the square root can also be rewritten with a continued fraction.

Proposition 1. The $\phi$-rotated Padé-type HABC (4) can be rewritten as the continued-fraction boundary condition (36) with $\alpha_{m}=e^{\imath \phi / 2}$ for $m=1 \ldots M$.

Proof. By injecting the formulation of the Padé approximation of the square root as a continued fraction in equation (3), we have successively

$$
\begin{aligned}
f_{2 N+1}^{\phi}(X) & =e^{\imath \phi / 2} f_{2 N+1}^{\text {Padé }}\left(e^{-\imath \phi}(1+X)-1\right) \\
& =e^{\imath \phi / 2}+e^{\imath \phi / 2} \frac{e^{-\imath \phi}(1+X)-1}{1+f_{2 N+1}^{\text {Padé }}\left(e^{-\imath \phi}(1+X)-1\right)} \\
& =e^{\imath \phi / 2}+\frac{X+1-e^{\ell \phi}}{e^{\imath \phi / 2}+f_{2 N+1}^{\phi}(X)} .
\end{aligned}
$$

It well known that the Higdon-type HABCs [36, 37, 40, 42, 43, 76] and the CRBCs [44] are related to rational approximations of the square root in the symbol of the exact DtN operator. These boundary conditions have been written with auxiliary fields verifying recursive relations, which are generally related to two sequences. In the first sequence, the auxiliary fields $\left\{u_{m}\right\}_{m=1 \ldots M}$ are governed by

$$
\left[\partial_{x}-\imath \kappa \alpha_{m}\right] u_{m}=u_{m-1}, \quad \text { for } m=1 \ldots M,
$$

with $u_{M}=u$ and $u_{0}=0$. In the second sequence, the auxiliary fields $\left\{u_{n}^{\star}\right\}_{n=1 \ldots N}$ verify

$$
\left[\partial_{x}-\imath \kappa \alpha_{2 n}\right] u_{n}^{\star}=\left[\partial_{x}+\imath \kappa \alpha_{2 n-1}\right] u_{n-1}^{\star}, \quad \text { for } n=1 \ldots N,
$$

with $u_{N}^{\star}=u$ and $u_{0}^{\star}=0$. In particular, the CRBC proposed in [44] is equivalent to a time-domain version of sequence (39) with the complex coefficients

$$
\alpha_{m}=\cos \theta_{m}\left(1+\frac{\tan ^{2} \theta_{m}}{-\imath \kappa c T}\right), \quad \text { for } m=1 \ldots M,
$$

where $\theta_{m}$ is an angle, $c$ is a velocity and $T$ is a time duration. The following propositions establish a formal link between these sequences and the continued-fraction boundary condition (36). Proposition 3 generalizes a result proved in [36, section 8.4].

Proposition 2. The continued-fraction boundary condition (36) can be rewritten with the sequence of auxiliary fields $\left\{u_{m}\right\}_{m=1 \ldots M}$ that verify equation (38). 
Proof. The proposition is proved by showing that

$$
\left[\partial_{x}-\imath \kappa f_{m}\left(\Delta_{\Gamma} / \kappa^{2}\right)\right] u_{m}=0, \quad \text { for } m=1 \ldots M,
$$

holds if the fields verify equation (38). We proceed by induction. For $m=1$, it is trivially true. For $m>1$, injecting equation (37) in equation (40) gives successively

$$
\begin{aligned}
& {\left[\partial_{x}-\imath \kappa \alpha_{m}-\imath \kappa \frac{\Delta_{\Gamma} / \kappa^{2}+1-\alpha_{m}^{2}}{\alpha_{m}+f_{m-1}\left(\Delta_{\Gamma} / \kappa^{2}\right)}\right] u_{m}=0} \\
& \Leftrightarrow \quad\left[-\imath \kappa\left(\Delta_{\Gamma} / \kappa^{2}+1\right)+\alpha_{m} \partial_{x}+f_{m-1}\left(\Delta_{\Gamma} / \kappa^{2}\right)\left[\partial_{x}-\imath \kappa \alpha_{m}\right]\right] u_{m}=0, \\
& \Leftrightarrow \quad\left[\partial_{x x}-\imath \kappa \alpha_{m} \partial_{x}-\imath \kappa f_{m-1}\left(\Delta_{\Gamma} / \kappa^{2}\right)\left[\partial_{x}-\imath \kappa \alpha_{m}\right]\right] u_{m}=0, \quad\left(\text { using } \Delta u_{m}+\kappa^{2} u_{m}=0\right) \\
& \Leftrightarrow \quad\left[\partial_{x}-\imath \kappa f_{m-1}\left(\Delta_{\Gamma} / \kappa^{2}\right)\right]\left[\partial_{x}-\imath \kappa \alpha_{m}\right] u_{m}=0, \\
& \Leftrightarrow \quad\left[\partial_{x}-\imath \kappa f_{m-1}\left(\Delta_{\Gamma} / \kappa^{2}\right)\right] u_{m-1}=0, \quad \text { (using (38)) }
\end{aligned}
$$

which is true if (40) holds for $m-1$.

Proposition 3. For $M=2 N-1$, the continued-fraction boundary condition (36) can be rewritten with the sequence of auxiliary fields $\left\{u_{n}^{\star}\right\}_{n=1 \ldots N}$ that verify equation (39). The auxiliary fields are related to those of the sequence (38) thanks to

$$
u_{n}^{\star}=\left[\prod_{i=n}^{N-1} \frac{1}{-\kappa^{2}\left(1+\Delta_{\Gamma} / \kappa^{2}\right)+\kappa^{2} \alpha_{2 i+1}^{2}}\right] u_{2 n} .
$$

Proof. For $m=1 \ldots M-1$, equation (38) can be successively written as

$$
\begin{aligned}
& {\left[\partial_{x}-\imath \kappa \alpha_{m}\right] u_{m}=u_{m-1},} \\
& \Leftrightarrow \quad\left[-\kappa^{2}\left(1+\Delta_{\Gamma} / \kappa^{2}\right)+\kappa^{2} \alpha_{m}^{2}\right] u_{m}=\left[\partial_{x}+\imath \kappa \alpha_{m}\right] u_{m-1}, \quad\left(\text { using } \Delta u_{m}+\kappa^{2} u_{m}=0\right) \\
& \left.\Leftrightarrow \quad\left[-\kappa^{2}\left(1+\Delta_{\Gamma} / \kappa^{2}\right)+\kappa^{2} \alpha_{m}^{2}\right]\left[\partial_{x}-\imath \kappa \alpha_{m+1}\right] u_{m+1}=\left[\partial_{x}+\imath \kappa \alpha_{m}\right] u_{m-1} . \quad \text { (using (38) with } m+1\right)
\end{aligned}
$$

Considering this equation for $m=2 n-1$ with $n=1 \ldots N$ gives

$$
\left[-\kappa^{2}\left(1+\Delta_{\Gamma} / \kappa^{2}\right)+\kappa^{2} \alpha_{2 n-1}^{2}\right]\left[\partial_{x}-\imath \kappa \alpha_{2 n}\right] u_{2 n}=\left[\partial_{x}+\imath \kappa \alpha_{2 n-1}\right] u_{2 n-2} .
$$

Defining the field $u_{n}^{\star}$ using the relation (41) leads to the sequence (39).

\section{B Variational formulation for the Helmholtz problem with HABC and corner treatment}

We consider the Helmholtz equation on a rectangular domain $\Omega$. The HABC is prescribed on every edge, and the corner treatment based on compatibility relations is used at every corner. The edges are denoted $\Gamma^{f}$ (with $f=1 \ldots 4$ ) and the corners are denoted $P^{f g}=\overline{\Gamma^{f}} \cap \overline{\Gamma^{g}}$ (where $\Gamma^{f}$ and $\Gamma^{g}$ are any adjacent edges). On each edge $\Gamma^{f}$, we define $N$ auxiliary fields $\left\{\varphi_{i}^{f}\right\}_{i=1 \ldots N}$. The variational formulation of the problem reads: Find $u \in H^{1}(\Omega)$ and $\varphi_{i}^{f} \in H^{1}\left(\Gamma^{f}\right)$, with $f=1 \ldots 4$ and $i=1 \ldots N$, such that

$$
\int_{\Omega}\left[\nabla u \cdot \nabla v-\kappa^{2} u v\right] d \Omega-\sum_{f=1}^{4} \int_{\Gamma^{f}} \mathcal{L}\left(u, \varphi_{1}^{f}, \ldots, \varphi_{N}^{f}\right) v d \Gamma=-\int_{\Omega} s v d \Omega, \quad \forall v \in H^{1}(\Omega),
$$

and

$$
\begin{aligned}
\int_{\Gamma^{f}}\left[\left(\partial_{\tau^{f}} \varphi_{i}^{f}\right)\left(\partial_{\tau^{f}} \rho^{f}\right)-\kappa^{2}\left(\left(e^{\imath \phi} c_{i}+1\right) \varphi_{i}^{f}+e^{\imath \phi}\left(c_{i}+1\right) u\right) \rho^{f}\right] d \Gamma & \\
- & \sum_{g}\left[\mathcal{L}\left(\varphi_{i}^{f}, \varphi_{i 1}^{f g}, \ldots, \varphi_{i N}^{f g}\right) \rho^{f}\right]_{P^{f g}}=0, \quad \forall \rho^{f} \in H^{1}\left(\Gamma^{f}\right),
\end{aligned}
$$

with $f=1 \ldots 4$ and $i=1 \ldots N$. In the last equation, the index $g$ corresponds to any edge $\Gamma^{g}$ adjacent to $\Gamma^{f}$. The linear algebraic operator $\mathcal{L}(\cdots)$ is defined in equation $(6)$. The variables $\varphi_{i j}^{f g}$ are defined as

$$
\varphi_{i j}^{f g}=-\frac{\left(c_{j}+1\right) \varphi_{i}^{f}+\left(c_{i}+1\right) \varphi_{j}^{g}}{c_{i}+c_{j}+e^{-\imath \phi}}, \quad \text { for } i, j=1 \ldots N, \quad \text { on } P^{f g} .
$$




\section{Acknowledgments}

This research was funded in part through the ARC grant for Concerted Research Actions (ARC WAVES 15/1903), financed by the Wallonia-Brussels Federation. The authors gratefully thanks the referees for their comments, which helped in significantly improving section 3.1.

\section{References}

[1] S. Acosta. High-order surface radiation conditions for time-harmonic waves in exterior domains. Computer Methods in Applied Mechanics and Engineering, 322:296-310, 2017.

[2] X. Antoine. Conditions de Radiation sur le Bord. PhD thesis, Université de Pau et des Pays de l'Adour, 1997.

[3] X. Antoine. Fast approximate computation of a time-harmonic scattered field using the on-surface radiation condition method. IMA Journal of Applied Mathematics, 66(1):83-110, 2001.

[4] X. Antoine. An algorithm coupling the OSRC and FEM for the computation of an approximate scattered acoustic field by a non-convex body. International Journal for Numerical Methods in Engineering, 54(7):1021-1041, 2002.

[5] X. Antoine, H. Barucq, and A. Bendali. Bayliss-Turkel-like radiation conditions on surfaces of arbitrary shape. Journal of Mathematical Analysis and Applications, 229(1):184-211, 1999.

[6] X. Antoine, A. Bendali, and M. Darbas. Analytic preconditioners for the boundary integral solution of the scattering of acoustic waves by open surfaces. Journal of Computational Acoustics, 13(03):477-498, 2005.

[7] X. Antoine and M. Darbas. Integral Equations and Iterative Schemes for Acoustic Scattering Problems. In F. Magoulès, editor, Numerical Methods for Acoustics Problems. Saxe-Coburg Editors, 2016.

[8] X. Antoine, M. Darbas, and Y. Y. Lu. An improved surface radiation condition for high-frequency acoustic scattering problems. Computer Methods in Applied Mechanics and Engineering, 195(33):4060-4074, 2006.

[9] X. Antoine and C. Geuzaine. Optimized Schwarz domain decomposition methods for scalar and vector Helmholtz equations. In D. Lahaye, J. Tang, and K. Vuik, editors, Modern Solvers for Helmholtz Problems, Lecture Notes in Geosystems Mathematics and Computing, pages 189-213. Birkauser, 2017.

[10] A. V. Astaneh and M. N. Guddati. A two-level domain decomposition method with accurate interface conditions for the Helmholtz problem. International Journal for Numerical Methods in Engineering, 107(1):74-90, 2016 .

[11] S. Asvadurov, V. Druskin, M. N. Guddati, and L. Knizhnerman. On optimal finite-difference approximation of PML. SIAM Journal on Numerical Analysis, 41(1):287-305, 2003.

[12] A. Bamberger, B. Engquist, L. Halpern, and P. Joly. Higher order paraxial wave equation approximations in heterogeneous media. SIAM Journal on Applied Mathematics, 48(1):129-154, 1988.

[13] A. Bamberger, P. Joly, and J. E. Roberts. Second-order absorbing boundary conditions for the wave equation: a solution for the corner problem. SIAM Journal on Numerical Analysis, 27(2):323-352, 1990.

[14] H. Barucq, A. Bendali, M. Fares, V. Mattesi, and S. Tordeux. A symmetric Trefftz-DG formulation based on a local boundary element method for the solution of the Helmholtz equation. Journal of Computational Physics, 330:1069-1092, 2017.

[15] H. Barucq, R. Djellouli, and A. Saint-Guirons. Three-dimensional approximate local DtN boundary conditions for prolate spheroid boundaries. Journal of Computational and Applied Mathematics, 234(6):1810-1816, 2010.

[16] H. Bériot, A. Prinn, and G. Gabard. Efficient implementation of high-order finite elements for Helmholtz problems. International Journal for Numerical Methods in Engineering, 106(3):213-240, 2016.

[17] A. Bermúdez, L. Hervella-Nieto, A. Prieto, R. Rodrı, et al. An optimal perfectly matched layer with unbounded absorbing function for time-harmonic acoustic scattering problems. Journal of Computational Physics, 223(2):469-488, 2007.

[18] Y. Boubendir, X. Antoine, and C. Geuzaine. A quasi-optimal non-overlapping domain decomposition algorithm for the Helmholtz equation. Journal of Computational Physics, 231(2):262-280, 2012.

[19] M. Cenanovic, P. Hansbo, and M. G. Larson. Finite element procedures for computing normals and mean curvature on triangulated surfaces and their use for mesh refinement. arXiv preprint arXiv:1703.05\%45, 2017.

[20] S. Chaillat, M. Darbas, and F. Le Louër. Approximate local Dirichlet-to-Neumann map for three-dimensional time-harmonic elastic waves. Computer Methods in Applied Mechanics and Engineering, 297:62-83, 2015.

[21] S. Chaillat, M. Darbas, and F. Le Louër. Fast iterative boundary element methods for high-frequency scattering problems in 3D elastodynamics. Journal of Computational Physics, 341:429-446, 2017. 
[22] S. N. Chandler-Wilde, D. P. Hewett, S. Langdon, and A. Twigger. A high frequency boundary element method for scattering by a class of nonconvex obstacles. Numerische Mathematik, 129(4):647-689, 2015.

[23] F. Collino. Conditions absorbantes d'ordre élevé pour des modèles de propagation d'onde dans des domaines rectangulaires. Technical Report 1790, INRIA, 1992.

[24] F. Collino. Conditions absorbantes d'ordre élevé pour les équations de maxwell dans des domaines rectangulaires. Technical Report 2932, INRIA, 1993.

[25] F. Collino. High order absorbing boundary conditions for wave propagation models. Straight line boundary and corner cases. In Second International Conference on Mathematical and Numerical Aspects of Wave Propagation (Newark, DE, 1993), pages 161-171, 1993.

[26] M. Darbas, E. Darrigrand, and Y. Lafranche. Combining analytic preconditioner and fast multipole method for the 3-D Helmholtz equation. Journal of Computational Physics, 236:289-316, 2013.

[27] E. Demaldent and S. Imperiale. Perfectly matched transmission problem with absorbing layers: Application to anisotropic acoustics in convex polygonal domains. International Journal for Numerical Methods in Engineering, 96(11):689-711, 2013.

[28] V. Druskin, S. Güttel, and L. Knizhnerman. Near-optimal perfectly matched layers for indefinite Helmholtz problems. SIAM Review, 58(1):90-116, 2016.

[29] P. Dular, C. Geuzaine, F. Henrotte, and W. Legros. A general environment for the treatment of discrete problems and its application to the finite element method. IEEE Transactions on Magnetics, 34(5):33953398, 1998.

[30] M. El Bouajaji, X. Antoine, and C. Geuzaine. Approximate local Magnetic-to-Electric surface operators for time-harmonic Maxwell's equations. Journal of Computational Physics, 279:241-260, 2014.

[31] B. Engquist and A. Majda. Absorbing boundary conditions for numerical simulation of waves. Proceedings of the National Academy of Sciences, 74(5):1765-1766, 1977.

[32] B. Engquist and A. Majda. Radiation boundary conditions for acoustic and elastic wave calculations. Communications on pure and applied mathematics, 32(3):313-357, 1979.

[33] T. Frankel. The Geometry of Physics: An Introduction. Cambridge University Press, 2011.

[34] M. J. Gander and H. Zhang. A class of iterative solvers for the Helmholtz equation: factorizations, sweeping preconditioners, source transfer, single-layer potentials, polarized traces, and optimized Schwarz methods. SIAM Review, 2018. To appear.

[35] C. Geuzaine and J.-F. Remacle. Gmsh: A 3-D finite element mesh generator with built-in pre-and postprocessing facilities. International Journal for Numerical Methods in Engineering, 79(11):1309-1331, 2009.

[36] D. Givoli, T. Hagstrom, and I. Patlashenko. Finite element formulation with high-order absorbing boundary conditions for time-dependent waves. Computer Methods in Applied Mechanics and Engineering, 195(29):3666-3690, 2006.

[37] D. Givoli and B. Neta. High-order non-reflecting boundary scheme for time-dependent waves. Journal of Computational Physics, 186(1):24-46, 2003.

[38] Z. Guan-Quan. High-order approximation of one-way wave equations. Journal of Computational Mathematics, 3:90-97, 1985.

[39] M. N. Guddati and K.-W. Lim. Continued-fraction absorbing boundary conditions for convex polygonal domains. International Journal for Numerical Methods in Engineering, 66(6):949-977, 2006.

[40] M. N. Guddati and J. L. Tassoulas. Continued-fraction absorbing boundary conditions for the wave equation. Journal of Computational Acoustics, 8(01):139-156, 2000.

[41] T. Hagstrom and S. Kim. Complete radiation boundary conditions for the Helmholtz equation I: waveguides. Numerische Mathematik, pages 1-50, 2019.

[42] T. Hagstrom, A. Mar-Or, and D. Givoli. High-order local absorbing conditions for the wave equation: Extensions and improvements. Journal of Computational Physics, 227(6):3322-3357, 2008.

[43] T. Hagstrom and T. Warburton. A new auxiliary variable formulation of high-order local radiation boundary conditions: corner compatibility conditions and extensions to first-order systems. Wave Motion, 39(4):327$338,2004$.

[44] T. Hagstrom and T. Warburton. Complete radiation boundary conditions: minimizing the long time error growth of local methods. SIAM Journal on Numerical Analysis, 47(5):3678-3704, 2009.

[45] T. Hagstrom, T. Warburton, and D. Givoli. Radiation boundary conditions for time-dependent waves based on complete plane wave expansions. Journal of Computational and Applied Mathematics, 234(6):1988-1995, 2010 .

[46] L. Halpern and L. N. Trefethen. Wide-angle one-way wave equations. The Journal of the Acoustical Society of America, 84(4):1397-1404, 1988.

[47] I. Harari and R. Djellouli. Analytical study of the effect of wave number on the performance of local 
absorbing boundary conditions for acoustic scattering. Applied Numerical Mathematics, 50(1):15-47, 2004.

[48] I. Harari, M. Slavutin, and E. Turkel. Analytical and numerical studies of a finite element PML for the Helmholtz equation. Journal of Computational Acoustics, 8(01):121-137, 2000.

[49] R. L. Higdon. Absorbing boundary conditions for difference approximations to the multidimensional wave equation. Mathematics of computation, 47(176):437-459, 1986.

[50] T. Huttunen, J. P. Kaipio, and P. Monk. The perfectly matched layer for the ultra-weak variational formulation of the 3D Helmholtz equation. International Journal for Numerical Methods in Engineering, 61(7):1072-1092, 2004.

[51] P. Joly, S. Lohrengel, and O. Vacus. Un résultat d'existence et d'unicité pour l'équation de Helmholtz avec conditions aux limites absorbantes d'ordre 2. Comptes Rendus de l'Académie des Sciences-Series IMathematics, 329(3):193-198, 1999.

[52] D. Justo, T. Warburton, and T. Hagstrom. Solving scattering problems for Maxwell's equations using polygonal artificial boundaries. In 7th International Conference on Mathematical and Numerical Aspects of Wave Propagation, pages 71-73, 2005.

[53] R. Kechroud, X. Antoine, and A. Soulaimani. Numerical accuracy of a Padé-type non-reflecting boundary condition for the finite element solution of acoustic scattering problems at high-frequency. International Journal for Numerical Methods in Engineering, 64(10):1275-1302, 2005.

[54] J. B. Keller and D. Givoli. Exact non-reflecting boundary conditions. Journal of Computational Physics, 82(1):172-192, 1989.

[55] T. Khajah, X. Antoine, and S. P. A. Bordas. B-Spline FEM for time-harmonic acoustic scattering and propagation. Journal of Theoretical and Computational Acoustics, 26(4):1850059, 2018.

[56] J. LaGrone and T. Hagstrom. Double absorbing boundaries for finite-difference time-domain electromagnetics. Journal of Computational Physics, 326:650-665, 2016.

[57] A. Lieu, G. Gabard, and H. Bériot. A comparison of high-order polynomial and wave-based methods for Helmholtz problems. Journal of Computational Physics, 321:105-125, 2016.

[58] E. L. Lindman. "Free-space" boundary conditions for the time-dependent wave equation. Journal of Computational Physics, 18(1):66-78, 1975.

[59] Y. Y. Lu. A complex coefficient rational approximation of $\sqrt{1+x}$. Applied numerical mathematics, 27(2):141-154, 1998.

[60] E. Magid, O. Soldea, and E. Rivlin. A comparison of Gaussian and mean curvature estimation methods on triangular meshes of range image data. Computer Vision and Image Understanding, 107(3):139-159, 2007.

[61] V. Mattesi, M. Darbas, and C. Geuzaine. A high-order absorbing boundary condition for 2D time-harmonic elastodynamic scattering problems. Computers $\&$ Mathematics with Applications, 2018. In press.

[62] M. Medvinsky, S. Tsynkov, and E. Turkel. Direct implementation of high order BGT artificial boundary conditions. Journal of Computational Physics, 376:98-128, 2019.

[63] F. A. Milinazzo, C. A. Zala, and G. H. Brooke. Rational square-root approximations for parabolic equation algorithms. The Journal of the Acoustical Society of America, 101(2):760-766, 1997.

[64] A. Modave, X. Antoine, and C. Geuzaine. An efficient domain decomposition method with cross-point treatment for Helmholtz problems. In 39th Ibero-Latin American Congress on Computational Methods in Engineering (Compiègne/Paris, France), pages 63-66, 2018.

[65] A. Modave, A. Atle, J. Chan, and T. Warburton. A GPU-accelerated nodal discontinuous Galerkin method with high-order absorbing boundary conditions and corner/edge compatibility. International Journal for Numerical Methods in Engineering, 112(11):1659-1686, 2017.

[66] A. Modave, E. Delhez, and C. Geuzaine. Optimizing perfectly matched layers in discrete contexts. International Journal for Numerical Methods in Engineering, 99(6):410-437, 2014.

[67] O. Ozgun and M. Kuzuoglu. Near-field performance analysis of locally-conformal perfectly matched absorbers via Monte-Carlo simulations. Journal of Computational Physics, 227(2):1225-1245, 2007.

[68] S. Petrides and L. F. Demkowicz. An adaptive DPG method for high frequency time-harmonic wave propagation problems. Computers \& Mathematics with Applications, 74(8):1999-2017, 2017.

[69] S. Savadatti and M. N. Guddati. A finite element alternative to infinite elements. Computer Methods in Applied Mechanics and Engineering, 199(33-36):2204-2223, 2010.

[70] K. Schmidt, J. Diaz, and C. Heier. Non-conforming Galerkin finite element methods for local absorbing boundary conditions of higher order. Computers 85 Mathematics with Applications, 70(9):2252-2269, 2015.

[71] K. C. Stein. Complete radiation boundary conditions: Corner and edge closure conditions. PhD thesis, Southern Methodist University, 2012.

[72] R. Tezaur, A. Macedo, C. Farhat, and R. Djellouli. Three-dimensional finite element calculations in acoustic scattering using arbitrarily shaped convex artificial boundaries. International Journal for Numerical Methods 
in Engineering, 53(6):1461-1476, 2002.

[73] E. Turkel. Boundary conditions and iterative schemes for the Helmholtz equation in unbounded regions. Computational Methods for Acoustics Problems, pages 127-158, 2008.

[74] E. Turkel and A. Yefet. Absorbing PML boundary layers for wave-like equations. Applied Numerical Mathematics, 27(4):533-557, 1998.

[75] O. Vacus. Mathematical analysis of absorbing boundary conditions for the wave equation: the corner problem. Mathematics of Computation, 74(249):177-200, 2005.

[76] V. J. Van Joolen, B. Neta, and D. Givoli. High-order Higdon-like boundary conditions for exterior transient wave problems. International Journal for Numerical Methods in Engineering, 63(7):1041-1068, 2005.

[77] L. Zepeda-Núñez and L. Demanet. Nested domain decomposition with polarized traces for the 2D Helmholtz equation. SIAM Journal on Scientific Computing, 40(3):B942-B981, 2018. 